\title{
iTRAQ-based proteomic profiling reveals protein alterations after traumatic brain injury and supports thyroxine as a potential treatment
}

\author{
Zhongxiang Zhang ${ }^{1,2}$, Jiangtao Yu ${ }^{1,2}$, Pengcheng Wang ${ }^{1,2}$, Lian Lin ${ }^{1,2}$, Ruining Liu ${ }^{1,2}$, Rong Zeng ${ }^{1,2}$, Haoli Ma ${ }^{2,3^{*}}$
} and Yan Zhao ${ }^{1,2^{*}}$

\begin{abstract}
Traumatic brain injury (TBI) is a primary cause of disability and death across the world. Previously, RNA analysis was widely used to study the pathophysiological mechanisms underlying TBl; however, the relatively low correlation between the transcriptome and proteome revealed that RNA transcription abundance does not reliably predict protein abundance, which led to the emergence of proteomic research. In this study, an iTRAQ proteomics approach was applied to detect protein alterations after TBI on a large scale. A total of 3937 proteins were identified, and 146 proteins were significantly changed after TBI. Moreover, 23 upregulated proteins were verified by parallel reaction monitoring (PRM), and fold changes in 16 proteins were consistent with iTRAQ outcomes. Transthyretin (Ttr) upregulation has been demonstrated at the transcriptional level, and this study further confirmed this at the protein level. After treatment with thyroxine (T4), which is transported by Ttr, the effects of T4 on neuronal histopathology and behavioral performance were determined in vivo (TBI+T4 group). Brain edema was alleviated, and the integrity of the blood brain barrier (BBB) improved. Escape latency in the Morris water maze (MWM) declined significantly compared with the group without T4 treatment. Modified neurological severity scores (mNSS) of the TBI +T4 group decreased from day 1 to day 7 post-TBI compared with the TBI + saline group. These results indicate that T4 treatment has potential to alleviate pathologic and behavioral abnormalities post-TBI. Protein alterations after T4 treatment were also detected by iTRAQ proteomics. Upregulation of proteins like Lgals3, Gfap and Apoe after TBI were reversed by T4 treatment. GO enrichment showed T4 mainly affected intermediate filament organization, cholesterol transportation and axonal regeneration. In summary, iTRAQ proteomics provides information about the impact of TBI on protein alterations and yields insight into underlying mechanisms and pathways involved in TBI and T4 treatment. Finally, Ttr and other proteins identified by iTRAQ may become potential novel treatment targets post-TBI.
\end{abstract}

Keywords: Traumatic brain injury, Quantitative proteomics, Mass spectrometry (MS), Parallel reaction monitoring (PRM), Thyroxine, Transthyretin, Rat cortex

\footnotetext{
*Correspondence: mahaoli@whu.edu.cn; doctoryanzhao@whu.edu.cn

${ }^{1}$ Emergency Center, Zhongnan Hospital of Wuhan University, Wuhan 430071, China

2 Hubei Clinical Research Center for Emergency and Resuscitation, Zhongnan Hospital of Wuhan University, Wuhan 430071, China

Full list of author information is available at the end of the article
}

\begin{abstract}
Introduction
Traumatic brain injury (TBI) is caused by an external force damaging the brain and is a major cause of disability and death in the United States [1]. In China, though the mortality rate due to TBI has declined in recent years, it remains relatively high, affecting 12.99 per 100,000 people in 2013 [2]. The economic burden of TBI is enormous, especially for people of low socioeconomic status, and these people happen to suffer
\end{abstract} permits use, sharing, adaptation, distribution and reproduction in any medium or format, as long as you give appropriate credit to the original author(s) and the source, provide a link to the Creative Commons licence, and indicate if changes were made. The images or other third party material in this article are included in the article's Creative Commons licence, unless indicated otherwise in a credit line to the material. If material is not included in the article's Creative Commons licence and your intended use is not permitted by statutory regulation or exceeds the permitted use, you will need to obtain permission directly from the copyright holder. To view a copy of this licence, visit http://creativecommons.org/licenses/by/4.0/. The Creative Commons Public Domain Dedication waiver (http://creativeco mmons.org/publicdomain/zero/1.0/) applies to the data made available in this article, unless otherwise stated in a credit line to the data. 
from TBI at a higher prevalence [3, 4]. The pathophysiology of TBI-related injury is divided into primary and secondary brain injury. Primary brain injury is caused directly by the impact. Secondary brain injury is characterized by a series of pathophysiological processes including electrolyte imbalance [5], mitochondrial dysfunction [6], neuroinflammation [7], brain edema [8], and cerebral vascular injury [9]. Despite some progress, the complexity and interactions within these pathways have not been fully illuminated. Thus, current treatment of TBI is still primarily based on symptomatic treatment, focusing on acute management instead of specific medicinal therapy targeting the pathways involved in post-TBI pathophysiology [10]. Hence, further research is required to elucidate the mechanisms underlying TBI and identify suitable therapeutic targets in order to determine more precise and potent clinical treatment methods.

Over the past few years, researchers have increasingly focused on complicated pathways underlying TBI and discovered some effective brain protection strategies. Previous research has revealed that the nuclear factorerythroid 2 related factor 2 (Nrf2) pathway protects TBI-affected rats from oxidative stress and TBI-induced apoptosis, and some pharmaceutical intervention targeting this pathway has been proven effective in animal experiments [11-14]. NF-kappa b-mediated signaling has been reported to have important associations with neuronal damage and neuroinflammation after TBI, and it may be a potential clinical target for some medications [15-18]. The PI3K/AKT pathway, associated with autophagy, also plays a vital role in neuronal protection from apoptosis after TBI [19-21]. Reducing JAK/ STAT pathway activation after experimental TBI has been reported to improve vestibular motor recovery via regulation of the $\gamma$-aminobutyric acid (GABA) type $\mathrm{A}$ receptor [22]. In addition, the mitogen-activated protein kinase (MAPK) pathway [23, 24], glycogen synthase kinase-3 (GSK3) pathway [25-27], and AMP-activated kinase (AMPK) pathway [28] are all considered to be possible therapeutic approaches.

Despite continuing efforts, the mechanisms involved in TBI are still not entirely clear. Currently, proteomics is popular in life science research to detect protein alterations and identify novel targets. Great advances in quantitative proteomics have been made, and proteomics has become large-scale and enabled detailed functional characterization of certain biochemical processes [29, 30]. Recent research has revealed that the average correlation between the transcriptome and proteome is less than $30 \%$, indicating that mRNA transcript abundance cannot reliably predict protein abundance. This inconsistency may be due to post-transcriptional modifications such as methylation and alternative splicing [31, 32], which makes proteomic research necessary.

A combination of liquid chromatography-tandem mass spectrometry (LC-MS/MS) and isobaric tags for relative and absolute quantitation (iTRAQ) has become an effective and widely used tool in quantitative proteomics studies, which is highly sensitive and repeatable [33]. Proteomic research on neural diseases has mainly focused on neurodegenerative diseases, such as Alzheimer's disease and Huntington's disease [34-36], epilepsy [37], narcolepsy [38], and ischemic cerebral disease [39, 40]. As for TBI, proteomics has been primarily applied to discover new biomarkers [41, 42]. In particular, Cheng et al. found that targeted temperature management (TTM) prevented TBI-induced neuronal necrosis, neuronal death, and brain edema, and some altered proteins (i.e., plasminogen, antithrombin III, and transthyretin) were identified [43]. Wu et al. detected protein changes in the traumatically injured hippocampus and found that pathways associated with global energy metabolism were significantly downregulated [44]. Song et al. determined that upregulation of the cAMP pathway was associated with mild TBI [45]. Overall, analyses of proteomic changes underlying TBI have been rarely reported. Particularly, results of the aforementioned research were not exactly consistent, because the samples were from different species and origins (Human blood, cells, hippocampus, brain tissue covering the injury or temporal globe in rats), and the labels were also different (iTRAQ or TMT). Besides, animals have individual differences. The different approaches like CCI, fluid induced percussion and blast were used for TBI models, which could also influence proteomic outcome. This study intends to detect the proteomic profile in cortex of rats and to identify key proteins involved in TBI pathophysiology.

In this study, LC-MS/MS combined with iTRAQ was utilized to identify differentially expressed proteins comparing rats in a TBI group with those in a sham group. Bioinformatic methods such as gene ontology (GO), the Kyoto Encyclopedia of Gene and Genomes (KEGG), and protein-protein interaction (PPI) analyses were used to analyze the biological processes, molecular functions, and interaction networks of these proteins. To confirm iTRAQ results, parallel reaction monitoring (PRM) was used to verify changes of proteins of interest. Using histopathology and behavioral measurements of the rats, the effects of thyroxine (T4), which is transported by transthyretin (Ttr), on pathophysiological features and neurological function after TBI were validated. This work may lead to a more comprehensive understanding of the mechanisms underlying TBI and the discovery of new pathways and molecules that can be used clinically. 


\section{Methods}

\section{Animals}

A total of 76 male Sprague-Dawley rats (aged 10 weeks, weighing 250-300 g) were purchased from Vital River Laboratory Animal Technology Co. Ltd. (Vital River, Beijing, China), kept at room temperature (RT) for a $12 \mathrm{~h}$ light/dark cycle, and had free access to food and water. The rats were kept in the Animal Experimental Center of Zhongnan Hospital of Wuhan University, China, for 1 week prior to surgery. All procedures were approved by the Animal Experiment Center and Ethics Committee of Zhongnan Hospital of Wuhan University and were performed under the National Institutes of Health Guide for the Care and Use of Laboratory Animals of China.

\section{Cortical contusion impact model and experimental groups}

The rats were randomly divided into two groups (sham group and TBI group), each of which contained 38 rats. A weight-drop device was applied to produce the cortical contusion impact (CCI), which was used to impose moderate TBI in previous studies $[46,47]$. Rats were anesthetized with $1 \%$ pentobarbital $(30 \mathrm{mg} / \mathrm{kg})$ by intraperitoneal (i.p.) injection. A midline scalp incision was then performed under sterile conditions. After exposing the skull, a hole (5 $\mathrm{mm}$ diameter) was drilled on the right, equidistant between the lambda and bregma $(2 \mathrm{~mm})$, exposing the dura mater. A $50 \mathrm{~g}$ weight fell vertically from a height of $25 \mathrm{~cm}$, striking the exposed brain tissue and causing moderate TBI. Rats in the sham group underwent identical surgical craniotomy without the contusion. Eight rats were divided into two groups (sham and TBI, $\mathrm{n}=4$ ). $24 \mathrm{~h}$ after surgery, the 8 rats were sacrificed and $3 \mathrm{~mm}$ of undamaged brain cortex around the damaged tissue was surgically collected for iTRAQ analysis. Another 8 rats were also divided into two groups (sham and TBI, $\mathrm{n}=4$ ) to verify the results of iTRAQ by PRM analysis. Moreover, another 12 rats (sham + saline, TBI + saline, and $\mathrm{TBI}+\mathrm{T} 4, \mathrm{n}=4$ ) were sacrificed and cortices were collected after behavioral experiments for iTRAQ analysis. The tissue for sequencing was stored in a refrigerator at $-80^{\circ} \mathrm{C}$.

\section{Protein extraction}

Cortical samples were transferred into low protein binding tubes (1.5 mL, Eppendorf, Hamburg, Germany) and lysed with $300 \mu \mathrm{L}$ of lysis buffer (Beyotime, Shanghai, China) and $1 \mathrm{mM}$ phenylmethyl sulfonyl fluoride (PMSF, Amresco, Solon, Ohio, USA). Next, the samples were homogenized and sonicated on ice to be lysed. The parameters were set to $1 \mathrm{~s}$ intervals $(3 \mathrm{~min}$ total) and a power of $80 \mathrm{~W}$. After being sonificated, the samples were centrifuged at $12,000 \times g$ for $10 \mathrm{~min}$ at RT, and supernatants were collected. Samples were centrifuged twice to exclude precipitation completely. Protein concentration was measured by bicinchoninic acid (BCA) assay (Thermo Fisher Scientific, Waltham, Massachusetts, USA), and aliquots were stored at $-80{ }^{\circ} \mathrm{C}$ for future analysis.

\section{Protein digestion}

The filter aided sample preparation (FASP) method was applied in order to enzymatically digest protein [48]. A total of $100 \mu \mathrm{g}$ of protein extraction was mixed with $120 \mu \mathrm{L}$ of reducing buffer $(\mathrm{pH}=8.0)$, containing $10 \mathrm{mM}$ dithiothreitol (DTT, Sangon Biotech, Shanghai, China), $8 \mathrm{M}$ urea, and $100 \mathrm{mM}$ tetraethylammonium bromide (TEAB, Sigma-Aldrich, St. Louis, Missouri, USA), in a $10 \mathrm{~K}$ ultrafiltration tube. The solution was incubated at $60{ }^{\circ} \mathrm{C}$ for $1 \mathrm{~h}$, and iodoacetamide (IAA, Sangon Biotech) was added in the dark at RT for 40 min to yield a final concentration of $50 \mathrm{mM}$. Afterward, solutions were centrifuged twice on the strainers at $12,000 \mathrm{rpm}$ for $20 \mathrm{~min}$ at $4{ }^{\circ} \mathrm{C}$, and liquid at the bottom was discarded. Then, $100 \mu \mathrm{L}$ of $300 \mathrm{mM}$ TEAB was added to every tube and centrifuged at $12,000 \mathrm{rpm}$ for $20 \mathrm{~min}$. The filter units were then transferred into new tubes with $100 \mu \mathrm{L}$ of $300 \mathrm{mM}$ TEAB. Subsequently, $3 \mu \mathrm{L}$ of $1 \mu \mathrm{g} / \mu \mathrm{L}$ sequencing-grade trypsin was added to solutions and incubated at $37{ }^{\circ} \mathrm{C}$ for $12 \mathrm{~h}$. Eventually, after digestion, peptides were collected and centrifuged at 12,000 rpm for $20 \mathrm{~min}$. Finally, $50 \mu \mathrm{L}$ of $200 \mathrm{mM}$ TEAB was added and centrifuged once.

\section{iTRAQ labeling}

After digestion, the lyophilized samples were resuspended in $100 \mu \mathrm{L}$ of $200 \mathrm{mM}$ TEAB, and one-fifth of each sample volume was transferred into a new collecting tube for labeling. Then, $200 \mu \mathrm{L}$ of isopropanol was added to iTRAQ reagent vial (AB SCIEX, Toronto, Canada) and centrifuged twice. Afterward, $100 \mu \mathrm{L}$ of iTRAQ label reagent was added to the samples and mixed. The mixture was incubated for $2 \mathrm{~h}$. Ultimately, $200 \mu \mathrm{L}$ of high-performance liquid chromatography (HPLC) water was added to the samples and incubated for $0.5 \mathrm{~h}$ for reaction termination. The labeled peptide solutions were freeze-dried in preparation for the next step.

\section{Reversed-Phase liquid chromatography (RPLC) analysis}

Reversed-phase separation was conducted on an 1100 HPLC System (Agilent, Santa Clara, California, USA), and an Agilent Zorbax Extend RP column (5 $\mu \mathrm{m}$, $150 \mathrm{~mm} \times 2.1 \mathrm{~mm})$ was applied. Mobile phases A $(2 \%$ acetonitrile in HPLC water) and B (90\% acetonitrile in HPLC water) were applied for RP gradient. The solvent gradient was set as follows: $0-8 \mathrm{~min}, 98 \% \mathrm{~A} ; 8.00 \mathrm{~min}$, 98-95\% A; 8-48 $\min , 95-75 \%$ A; 48-60 min, 75-60\% A; 
$60 \mathrm{~min}, 60-10 \% \mathrm{~A} ; 60-70 \mathrm{~min}, 10 \%$ A; $70 \mathrm{~min}, 10-98 \%$ A; 70-75 min, 98\% A. Tryptic peptides were separated at a velocity of $300 \mu \mathrm{L} / \mathrm{min}$. Monitoring was at $210 \mathrm{~nm}$ and $280 \mathrm{~nm}$. Samples were collected after $8 \mathrm{~min}$ and up to $60 \mathrm{~min}$, and elution buffer was harvested every $1 \mathrm{~min}$ into centrifuge tubes. The separated peptides were lyophilized in a vacuum.

\section{Mass spectrometry}

All analyses were conducted by a Q-Exactive mass spectrometer (Thermo Fisher Scientific). Samples were separated by a chromatographic column (PepMap C18, $100 \AA$, Thermo Fisher). The flow rate was set to $300 \mathrm{~nL} / \mathrm{min}$, and the gradient was $60 \mathrm{~min}(0-40 \mathrm{~min}, 5-30 \% \mathrm{~B} ; 40-54 \mathrm{~min}$, 30-50\% B; 54-55 min, 50-100\% B; 55-60 min, 100\% B; mobile phase $A=0.1 \% \mathrm{FA}$ in water and phase $\mathrm{B}=80 \%$ $\mathrm{ACN} / 0.1 \% \mathrm{FA}$ in water).

Complete mass spectrometry (MS) scans were obtained in the mass range of $300-1600 \mathrm{~m} / \mathrm{z}$ with a resolution of 35,000 . The target value of automatic gain control (AGC) was 1e6. The 10 most intense peaks were fragmented using higher energy collisional dissociation (HCD). Normalized collision energy (NCE) was set to 30. MS/MS spectra were acquired with a resolution of 17,500 , the AGC target was 2e5, and the maximum injection time was $50 \mathrm{~ms}$. Q-Exact dynamic exclusion was set to $30 \mathrm{~s}$ and ran in positive mode.

\section{Proteomic data analysis}

The resulting data were searched in the UniProt database, utilizing the Rattus norvegicus (Rat) taxonomy (https:// www.uniprot.org/proteomes/UP000002494). Data were analyzed by Proteome Discoverer ${ }^{\mathrm{TM}} 2.2$ (Thermo Fisher) software. The false discovery rate (FDR) of peptide search was controlled below $1 \%$. At least one unique peptide was required to qualify a protein. Missed cleavage was set at 2. MS1 tolerant was set at $10 \mathrm{ppm}$ and MS2 tolerant was $0.02 \mathrm{Da}$. The fixed modifications of iTRAQ 8-plex (N-term, K, Y), Carbamidomethyl (C) were specified.

Gene ontology enrichment (http://www.geneontolo gy.org) and KEGG (http://www.genome.jp/kegg) pathway analyses of altered proteins were conducted by $\mathrm{R}$ based on the hypergeometric distribution.

\section{Parallel reaction monitoring}

After extraction and digestion, proteins from eight samples were used for PRM ( $n=4$ per group). The immunoreactive trypsin (iRT) standard (Biognosys, Thermo Fisher) was dissolved to $10 \times$ and stored at $4{ }^{\circ} \mathrm{C}$. The $10 \times$ iRT standard peptide mix was added to peptide samples before LC-MS injection. The ratio of iRT to samples was set at $1: 10 \mathrm{v} / \mathrm{v}$.
The samples were fractionated on an Agilent 1100 liquid chromatograph $(\mathrm{pH}=10)$. Ultimately, six fractions were collected and run in data-dependent acquisition (DDA) mode. In short, the DDA raw files were searched on the database. In this database, the iRT peptide sequences with 11 entries were added with ProteomeDiscover (version 2.3). Fixed modifications were set to carbamidomethylation of cysteine, and variable modifications were set to oxidation of methionine and acetyl (protein $\mathrm{N}$ terminus). A maximum of two missed cleavages were permitted. The identifications were filtered to acquire $1 \%$ FDR at the peptide and protein levels. A list of peptides from DDA analysis was made for PRM validation (at least two peptides per protein). The flow rate was set to $300 \mathrm{~nL} / \mathrm{min}$. For PRM, precursors were targeted in a $1.2 \mathrm{~m} / \mathrm{z}$ isolation window around the $\mathrm{m} / \mathrm{z}$ of interest. Precursors were fragmented in HCD mode, and NCE energy was set to 32. MS/MS was performed at 30,000 resolution, and an AGC target of 5e5 spectra was manually verified using Skyline. The quantitative data were exported to an Excel spreadsheet, and the data were normalized to the total ion currant (TIC) of the MS runs.

\section{Levothyroxine treatment}

Levothyroxine (MCE, HY-18341) is a synthetic form of T4 (referred to here as T4). T4 was infused $1 \mathrm{~h}$ after CCI. Rats were randomly divided into four groups: sham + saline, sham $+\mathrm{T} 4, \mathrm{TBI}+$ saline, and $\mathrm{TBI}+\mathrm{T} 4$ ( $n=15$ per group). A total of $100 \mu \mathrm{g}$ of T4 was dissolved in $10 \mathrm{~mL}$ of saline and used for i.p. injection. The sham $+\mathrm{T} 4$ and $\mathrm{TBI}+\mathrm{T} 4$ groups received $\mathrm{T} 4$ at a concentration of $2.5 \mu \mathrm{g}$ per $100 \mathrm{~g}$ of weight. The injection dose, treatment window, and method of delivery were set based on previous research [49-51].

\section{Modified neurological severity score}

The modified neurological severity score (mNSS) was measured on days $1,3,5$, and 7 post-CCI to evaluate the neurological function of the rats ( $n=6$ per group). The mNSS consists of motor, sensory, balance, and reflex tests. The highest scores of the four tests are 6, 2, 6, and 4 , respectively. A higher score represents a more serious neurological deficit.

\section{Morris water maze}

The Morris water maze (MWM) was used to test spatial learning ability and memory in rats ( $n=6$ per group). Briefly, from days 7 to 11 post-CCI, the rats were placed in a circular tank with water containing of Soft Gel Paste (AmeriColor, Placentia, California, USA) and trained to find an underwater platform. Four training sessions per day were conducted. On the first day, rats were placed from the first quadrant to the fourth quadrant, whereas 
on the second day, training started from the second quadrant to first quadrant, and on the third day, training started from the third quadrant to the second quadrant, and so on. Escape latency was recorded, and the mean time of four trainings on each day was calculated. On the test day (day 12), the platform was removed, and the rats were placed opposite from the platform quadrant. After a $120 \mathrm{~s}$ test, the escape latency, time spent in the platform quadrant, and number of times crossing the platform area were recorded by the automatic tracking system (Xmaze ${ }^{\mathrm{TM}}$, Xinruan Information Technology Co., Shanghai, China). Training and testing were performed by blinded researchers.

\section{Cresyl violet staining and lesion volume assessment}

At $24 \mathrm{~h}$ post-CCI, rats ( $\mathrm{n}=3$ per group) were anesthetized, and hearts were exposed by thoracotomy. Fifty milliliter of saline was infused to flush the blood, and $100 \mathrm{~mL}$ of $4 \%$ paraformaldehyde was injected for perfusion fixation. After perfusion, brains were removed and placed in paraformaldehyde at $4{ }^{\circ} \mathrm{C}$ for $24 \mathrm{~h}$. After paraffin embedding, three 2-mm-thick coronal sections spanning across $6 \mathrm{~mm}$ to cover the whole damaged area were sliced and stained with cresyl violet (Solarbio, Beijing, China). Tissue volume loss was calculated by subtracting the ipsilateral volume (damaged hemisphere) from the contralateral volume (controlled hemisphere) [43]. The volumes of substructures were calculated by multiplying the area by section thickness. ImageJ software was applied to calculate the areas of substructures.

\section{Measurement of brain water content}

At $24 \mathrm{~h}$ post-CCI, a wet-to-dry weight ratio was applied to reflect the degree of cerebral edema ( $n=3$ per group) [52]. After sacrifice, the brain parenchyma was extracted, and the cerebellum and olfactory bulb were discarded. The parenchyma was divided into two hemispheres: contralateral (control) and ipsilateral (damaged). Samples were weighed to acquire a wet weight immediately, then dried at $110^{\circ} \mathrm{C}$ in a drying oven for $24 \mathrm{~h}$ to acquire a dry weight.

\section{Evaluation of blood-brain barrier (BBB) integrity}

Rats were anesthetized $23 \mathrm{~h}$ post-CCI, and $2 \%$ Evans blue (EB) dye was injected through the caudal vein at a dose of $4 \mathrm{~mL} / \mathrm{kg}$ and circulated for $1 \mathrm{~h}$ before sacrifice ( $\mathrm{n}=3$ per group). The hearts were perfused with saline through the left ventricle until colorless perfusion was obtained. Afterwards, rats were sacrificed, and brains were removed and weighed. After homogenizing in $3 \mu \mathrm{L} /$ mg formamide, samples were stored at RT for $48 \mathrm{~h}$. Following centrifugation, the supernatant was collected, and the optical density was measured at $625 \mathrm{~nm}$ to detect the relative amount of EB.

\section{Statistical analysis}

The Gaussian distribution for the quantitative proteomics data was analyzed using SPSS software (version 22.0, SPSS Chicago, Illinoi, USA) and Prism (version 8.0, San Diego, California, USA). Except proteomics and PRM data, other data were expressed as means \pm standard deviation (SD). Student's $t$-test was used for data analysis. A 2-tailed $\mathrm{p}$ value less than 0.05 was considered significant.

\section{Results \\ ITRAQ analysis of differentially expressed proteins}

Eight brain cortical samples were isolated from two groups and analyzed using iTRAQ label with LC-MS/ MS (Fig. 1). Principle component analysis (PCA) was performed to compare proteomes between the TBI and sham groups, and it was observed that the groups of samples were well-clustered (Fig. 2a). Based on the discovery analysis, a total of 3937 proteins were identified and quantified, of which 125 proteins were upregulated and 21 proteins were downregulated significantly (fold change $>1.20$ and $<5 / 6, p<0.05$ as threshold; Fig. 2b; Table 1). The top five proteins based on the fold change included Hspa4, Alb, Hba1, Hbb, and Krt42. The top five proteins based on the $p$-value included Krt10, S100a8, Mug1, Hpx, and Gc (Fig. 2c). Hierarchical clustering analysis (heat map) was generated to show differential protein patterns among samples and groups. As shown in Fig. 2d, two groups showed disparate protein change patterns, and most of the samples within one group were upregulated or downregulated consistently, which indicated good reproducibility. Additional file 1: Table S1 shows raw data of all identified proteins and differentially expressed proteins by iTRAQ.

\section{Gene ontology analysis}

By GO analysis, the biological process (BP), cellular component (CC), and molecular function (MF) of genes in different proteins were annotated. As Fig. 3a shows, the most overrepresented biological processes were related to regulation of endopeptidase, hydrolase, and peptidase, as well as negative regulation of catalytic activity and proteolysis. In CC annotation, the highly enriched terms included the extracellular region, extracellular space, blood microparticle, extracellular vesicle, extracellular organelles, and extracellular exosome (Fig. 3b). From this, it can be inferred that most differentially expressed proteins were extracellular, including Ttr. The molecular function annotation was consistent with BP, including inhibition of enzymes such as endopeptidase 


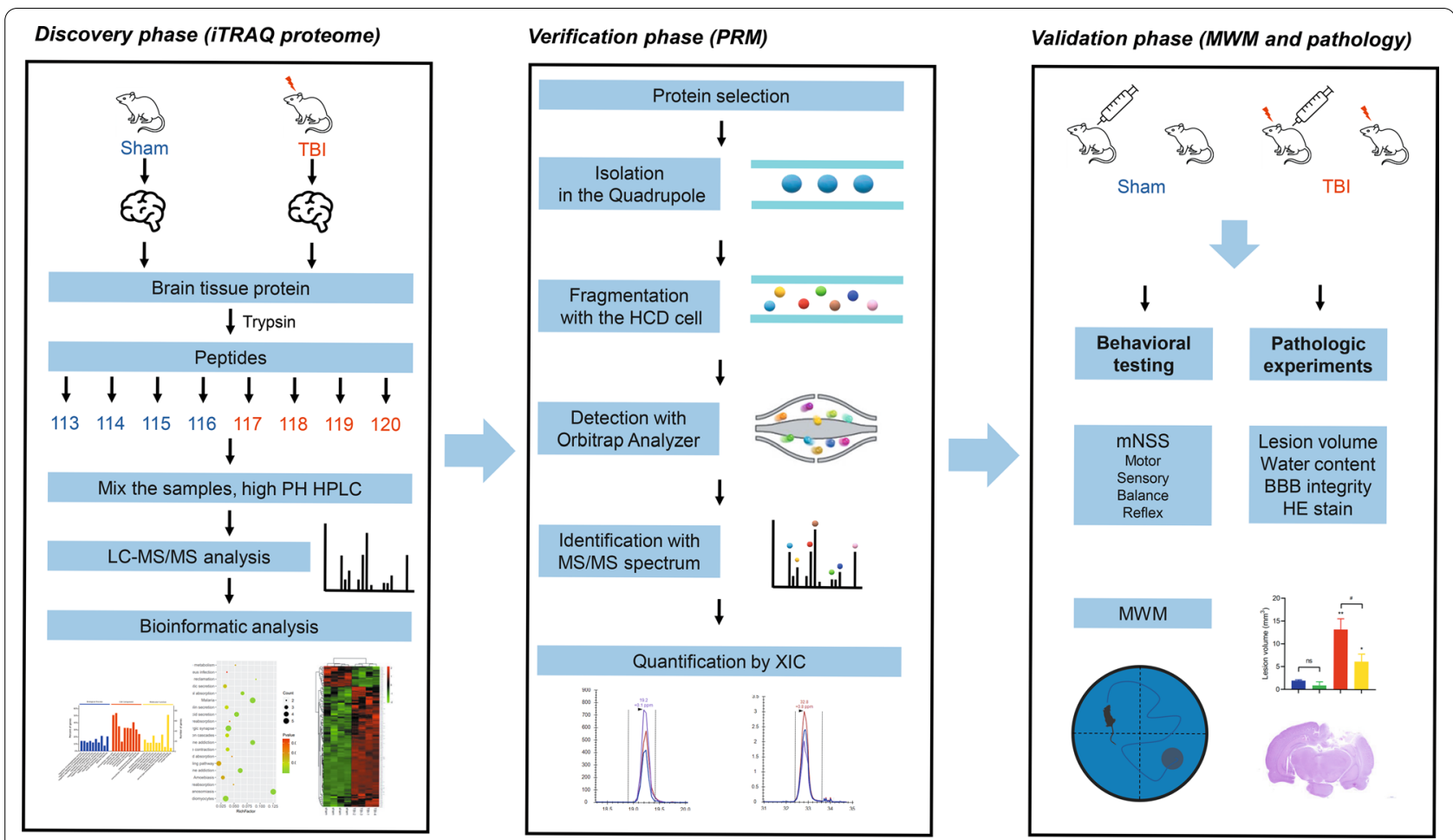

Fig. 1 Systematic workflow of three phases in the study. In the discovery phase, 8-plex iTRAQ was conducted, and bioinformatic analysis was performed. In the verification phase, PRM was applied to verify the outcomes of quantitative proteomic analysis. In the validation phase, a protein of interest (transthyretin) was identified, and thyroxine was used to detect the effects. Behavioral outcomes and histopathology were observed, and conclusions were made

and peptidase. Some proteins were also related to protein binding (i.e., Ttr and Camk2g) and oxygen binding (i.e., Alb, Hbb, and Hba1; Fig. 3c).

\section{KEGG analysis}

To reveal the pathways involved in proteins that were differentially expressed between TBI and sham groups, KEGG pathway analysis was employed. As shown in Fig. 3d, the most significantly enriched pathway, and with the highest rich factor, was African trypanosomiasis. Hemoglobin-related proteins such as Hbb, Hba1, Hba-a3, and ApoA1 were enriched in this pathway. The malaria pathway is similar to African trypanosomiasis, as proteins associated with this pathway also all originated from blood. Cocaine addiction, amphetamine addiction, and dopaminergic synapses were also significant pathways and shared altered proteins such as protein phosphatase 1 regulatory subunit 1B (Ppp1r1b), tyrosine hydroxylase (Th), and DOPA decarboxylase (Ddc), which are all associated with dopamine synthesis, except for calcium/ calmodulin-dependent protein kinase type II subunit gamma (Camk2g), which is thought to be a mediator of memory by playing an important role in memory destabilization [53]. Other pathways with top 20 enrichment score values included gastric acid secretion, adrenergic signaling in cardiomyocytes, mineral absorption, proximal tubule bicarbonate reclamation, cardiac muscle contraction, complement and coagulation cascades, insulin secretion, pancreatic secretion, amoebiasis, tyrosine metabolism, cAMP signaling pathway, aldosterone-regulated sodium reabsorption, carbohydrate digestion and absorption, endocrine and other factor-regulated calcium reabsorption, and Staphylococcus aureus infection.

\section{Protein-protein interaction analysis}

In order to further comprehend the interactions among the altered proteins, bioinformatics analysis (in this case, PPI) was performed. According to Fig. 3e, upregulated proteins (Ppp1r1b, Th, and Ddc) and one downregulated protein (Camk2g) were more relevant to cocaine addiction and amphetamine addiction pathways. Interestingly, proteins associated with myocardium-related pathways and gastric acid secretion pathways were all downregulated (i.e., Atp1a2, Atp1a1, Camk2g, Kcnj10, and Cacng3).

As Fig. 3e shows, the following proteins were identified: Ttr interacting with Tf (serotransferrin), ApoA1, Hp (haptoglobin), Itih4 (inter-alpha-trypsin inhibitor, 

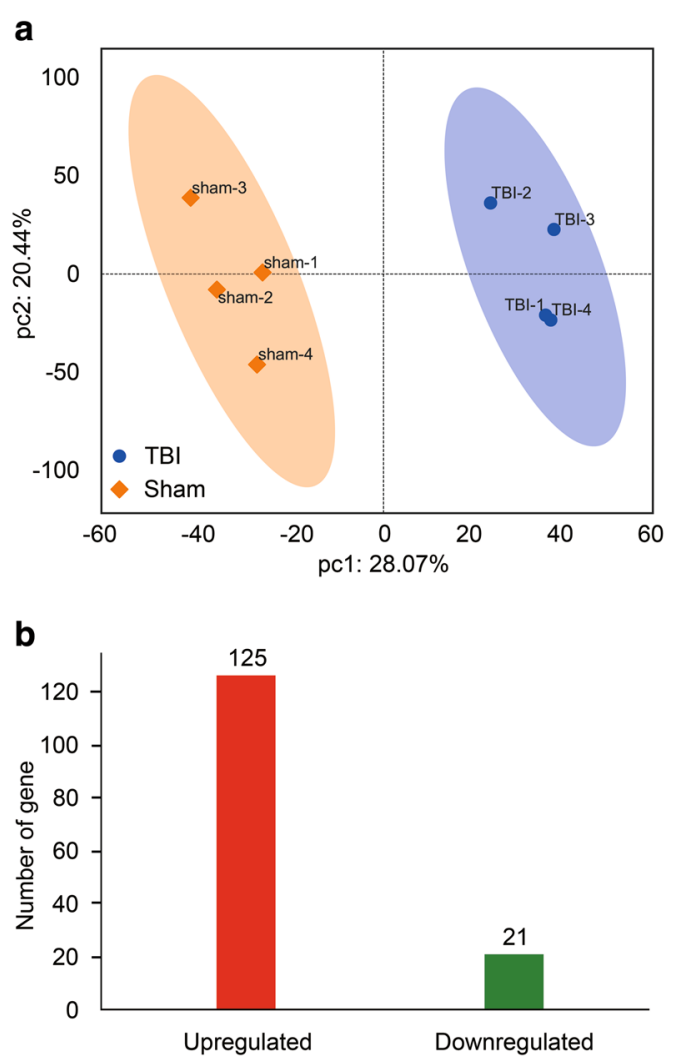

\section{d}
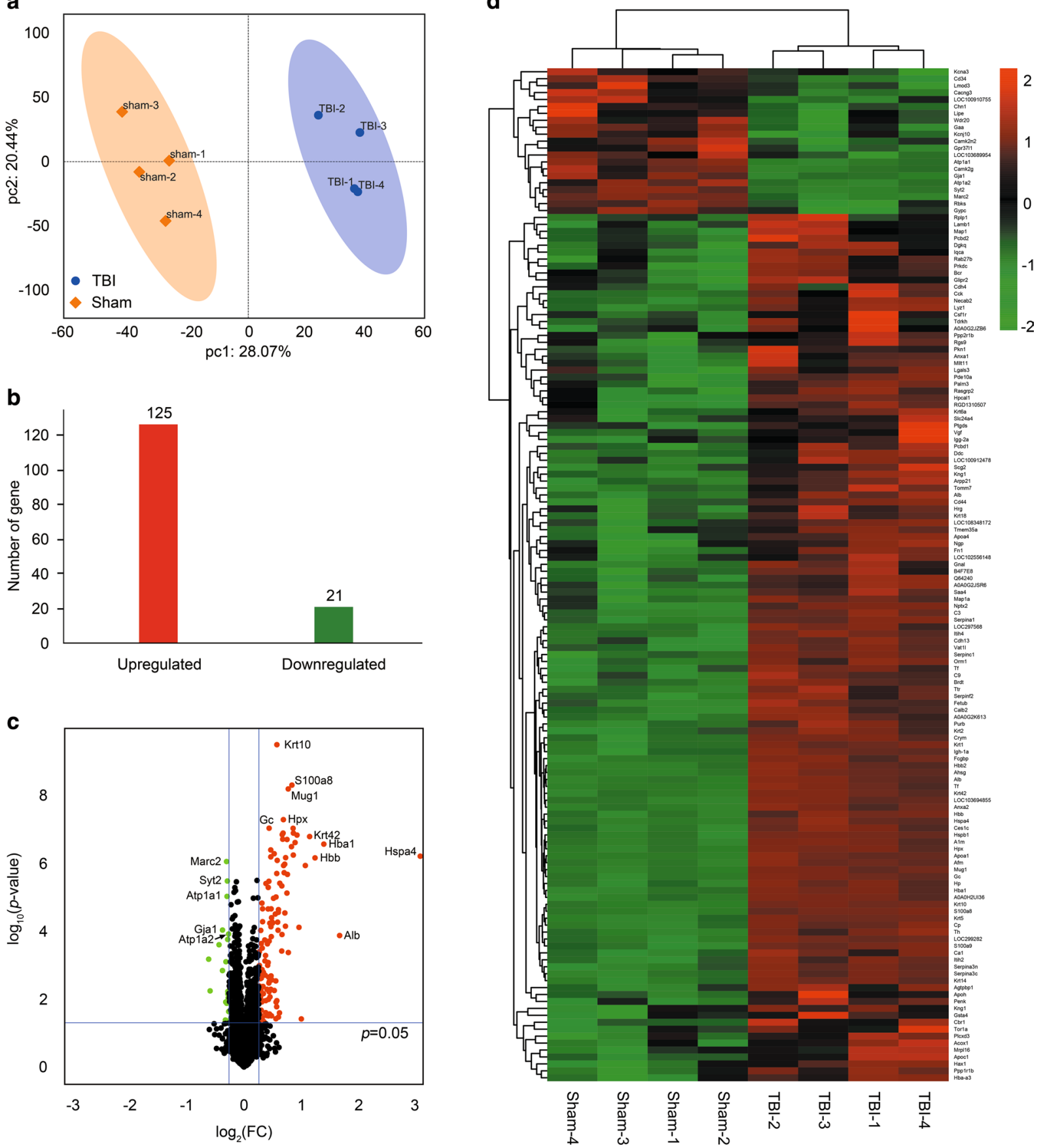

Fig. 2 General proteomic outcomes. a Principal component analysis indicated a near complete separation of proteins in the TBI (blue) and sham (yellow) groups. b 125 proteins were upregulated, and 21 proteins were downregulated. c Volcano plot showed differentially expressed proteins. The red and green dots indicate significantly upregulated and downregulated proteins, respectively. $\mathbf{d}$ Hierarchical cluster analysis. Red indicates upregulation, and green indicates downregulation. The dendrograms represent the classification of proteins. The number in the color scale indicates z-score

heavy chain 4), Afm (afamin), and lysozyme C-1. Ambp (alpha-1-microglobulin/bikunin precursor) interacted with A1m (alpha-1-microglobulin), Hpx (hemopexin), Gc
(GC vitamin D binding protein), Ahsg (alpha 2-HS glycoprotein), ApoA1, Apoh, Itih4, Itih2, Afm, C9, and Orm1 (orosomucoid 1). Th interacted with Ddc, Pcbd1 (pterin-4 
Table 1 Differentially expressed proteins after TBI by iTRAQ

\begin{tabular}{|c|c|c|c|c|c|c|c|}
\hline \multirow[t]{2}{*}{ Accession } & \multirow[t]{2}{*}{ Description } & \multirow[t]{2}{*}{ Coverage (\%) } & \multirow[t]{2}{*}{ MW (kDa) } & \multicolumn{2}{|c|}{ Average } & \multirow[t]{2}{*}{ p-value } & \multirow[t]{2}{*}{$\mathrm{FC}$} \\
\hline & & & & Sham & TBI & & \\
\hline D3ZVU4 & Ribokinase (Rbks) & 11 & 34 & 121.2 & 78.8 & 0.00070 & 0.65 \\
\hline P30337 & N-chimaerin (Chn1) & 4 & 38.2 & 120.4 & 79.7 & 0.0057 & 0.66 \\
\hline B1PLB1 & CD34 antigen (Cd34) & 11 & 41.2 & 115.1 & 84.9 & 0.00030 & 0.74 \\
\hline Q6XFR6 & Glycophorin-C (Gypc) & 27 & 10.4 & 113.0 & 87.0 & 0.0014 & 0.77 \\
\hline P08050 & Gap junction alpha-1 protein (Gja1) & 17 & 43 & 113.0 & 87.0 & 0.00010 & 0.77 \\
\hline P15384 & Potassium voltage-gated channel subfamily A member 3 (Kcna3) & 8 & 58.4 & 111.3 & 88.7 & 0.042 & 0.80 \\
\hline P49655 & ATP-sensitive inward rectifier potassium channel 10 (Kcnj10) & 4 & 42.5 & 111.1 & 88.9 & 0.012 & 0.80 \\
\hline Q6P7A9 & Lysosomal alpha-glucosidase (Gaa) & 4 & 106.1 & 110.9 & 89.0 & 0.00080 & 0.80 \\
\hline D3Z900 & Mitochondrial amidoxime reducing component 2 (Marc2) & 16 & 38.2 & 110.6 & 89.4 & $<0.0001$ & 0.81 \\
\hline D4A871 & Leiomodin 3 (Lmod3) & 2 & 66 & 110.5 & 89.5 & 0.013 & 0.81 \\
\hline P06685 & Sodium/potassium-transporting ATPase subunit alpha-1 (Atp1a1) & 46 & 113 & 110.3 & 89.7 & $<0.0001$ & 0.81 \\
\hline D3ZSW9 & RUN and FYVE domain-containing 2 (LOC100910755) & 2 & 69.9 & 110.3 & 89.8 & 0.013 & 0.81 \\
\hline P29101 & Synaptotagmin-2 (Syt2) & 31 & 47.2 & 110.1 & 89.9 & $<0.0001$ & 0.82 \\
\hline P11730 & $\begin{array}{l}\text { Calcium/calmodulin-dependent protein kinase type II subunit gamma } \\
\text { (Camk2g) }\end{array}$ & 28 & 59 & 109.9 & 90.1 & 0.00020 & 0.82 \\
\hline B4F7C1 & G protein-coupled receptor 37-like 1, isoform CRA_a (Gpr3711) & 2 & 52.8 & 109.8 & 90.2 & 0.0060 & 0.82 \\
\hline D4A617 & Ectonucleoside triphosphate diphosphohydrolase 1 (LOC103689954) & 3 & 52 & 109.8 & 90.2 & 0.0071 & 0.82 \\
\hline Q8VHX0 & Voltage-dependent calcium channel gamma-3 subunit (Cacng3) & 10 & 35.5 & 109.6 & 90.4 & 0.0093 & 0.82 \\
\hline P06686 & Sodium/potassium-transporting ATPase subunit alpha-2 (Atp1a2) & 45 & 112.1 & 109.4 & 90.7 & 0.00010 & 0.83 \\
\hline AOAOG2K2B7 & WD repeat domain 20 (Wdr20) & 4 & 64.6 & 109.3 & 90.7 & 0.023 & 0.83 \\
\hline G3V8R5 & Hormone-sensitive lipase (Lipe) & 1 & 116.8 & 109.3 & 90.8 & 0.044 & 0.83 \\
\hline Q9Z2N6 & Calcium/calmodulin-dependent protein kinase II inhibitor 2 (Camk2n2) & 16 & 8.6 & 109.1 & 90.9 & 0.0095 & 0.83 \\
\hline P20760 & Ig gamma-2A chain C region (lgg-2a) & 16 & 35.2 & 90.8 & 109.3 & 0.022 & 1.20 \\
\hline Q9QYJ6 & $\begin{array}{l}\text { CAMP and cAMP-inhibited cGMP 3',5'-cyclic phosphodiesterase 10A } \\
\text { (Pde10a) }\end{array}$ & 14 & 90.1 & 90.7 & 109.4 & 0.00040 & 1.21 \\
\hline D3ZMR1 & Translocase of outer mitochondrial membrane 7 (Tomm7) & 11 & 6.2 & 90.6 & 109.4 & 0.0010 & 1.21 \\
\hline P14173 & Aromatic-L-amino-acid decarboxylase (Ddc) & 11 & 54 & 90.6 & 109.4 & 0.0042 & 1.21 \\
\hline Q99P74 & Ras-related protein Rab-27B (Rab27b) & 5 & 24.6 & 90.6 & 109.4 & 0.0062 & 1.21 \\
\hline MORDW3 & N-acetyltransferase 8 (GCN5-related) family member 4 (LOC102556148) & 3 & 25.6 & 90.4 & 109.6 & 0.0079 & 1.21 \\
\hline D3ZM03 & $\begin{array}{l}\text { Vacuolar ATPase assembly integral membrane protein VMA21 } \\
\text { (LOC100912478) }\end{array}$ & 12 & 11.4 & 90.3 & 109.8 & 0.0044 & 1.22 \\
\hline D3ZCC3 & Slc24a4 protein (Slc24a4) & 4 & 62.8 & 90.3 & 109.8 & 0.045 & 1.22 \\
\hline P14942 & Glutathione S-transferase alpha-4 (Gsta4) & 8 & 25.5 & 90.1 & 110.0 & 0.043 & 1.22 \\
\hline R9PXW6 & RAS guanyl releasing protein 2, isoform CRA_b (Rasgrp2) & 5 & 69.3 & 90.0 & 110.0 & 0.0025 & 1.22 \\
\hline F1LXF1 & BCR, RhoGEF and GTPase-activating protein (Bcr) & 6 & 120 & 90.0 & 110.0 & 0.0088 & 1.22 \\
\hline Q4QQT4 & $\begin{array}{l}\text { Serine/threonine-protein phosphatase } 2 \mathrm{~A} 65 \mathrm{kDa} \text { regulatory subunit A } \\
\text { beta isoform (Ppp2r1b) }\end{array}$ & 11 & 66 & 89.8 & 110.2 & 0.046 & 1.23 \\
\hline Q5FVI0 & Arpp-21 protein (Arpp21) & 34 & 9.6 & 89.8 & 110.2 & 0.00010 & 1.23 \\
\hline Q6JAM9 & Transmembrane protein 35A (Tmem35a) & 5 & 18.5 & 89.8 & 110.3 & 0.0054 & 1.23 \\
\hline D3ZEY4 & Diacylglycerol kinase (Dgkq) & 3 & 102.5 & 89.7 & 110.3 & 0.0078 & 1.23 \\
\hline A0A0G2JXN6 & Galectin (Lgals3) & 4 & 27.6 & 89.7 & 110.3 & 0.031 & 1.23 \\
\hline $\mathrm{D} 4 \mathrm{~A} 1 \mathrm{H} 2$ & $\begin{array}{l}\text { Phosphatidylinositol-specific phospholipase } \mathrm{C}, \mathrm{X} \text { domain-containing } 3 \\
\text { (Plcxd3) }\end{array}$ & 8 & 36.3 & 89.5 & 110.5 & 0.021 & 1.23 \\
\hline F1LQY6 & $\mathrm{N}$-terminal EF-hand calcium-binding protein 2 (Necab2) & 16 & 43.5 & 89.5 & 110.5 & 0.00020 & 1.23 \\
\hline F1LST1 & Fibronectin (Fn1) & 4 & 262.6 & 89.5 & 110.5 & 0.0024 & 1.24 \\
\hline D4A1J3 & Paralemmin 3 (Palm3) & 7 & 78.5 & 89.3 & 110.7 & 0.0020 & 1.24 \\
\hline Q5M971 & Protein AF1q (MIlt11) & 8 & 10 & 89.2 & 110.8 & 0.012 & 1.24 \\
\hline Q5M7T5 & $\begin{array}{l}\text { Serine (Or cysteine) peptidase inhibitor, clade C (Antithrombin), member } \\
1 \text { (Serpinc } 1 \text { ) }\end{array}$ & 16 & 52.2 & 89.1 & 110.9 & $<0.0001$ & 1.24 \\
\hline A0A0G2K896 & Similar to RIKEN cDNA 1300017J02 (RGD1310507) & 5 & 76.7 & 89.1 & 111.0 & 0.0014 & 1.25 \\
\hline
\end{tabular}


Table 1 (continued)

\begin{tabular}{|c|c|c|c|c|c|c|c|}
\hline \multirow[t]{2}{*}{ Accession } & \multirow[t]{2}{*}{ Description } & \multirow[t]{2}{*}{ Coverage (\%) } & \multirow[t]{2}{*}{ MW (kDa) } & \multicolumn{2}{|c|}{ Average } & \multirow[t]{2}{*}{$p$-value } & \multirow[t]{2}{*}{$\mathrm{FC}$} \\
\hline & & & & Sham & TBI & & \\
\hline P34926 & $\begin{array}{l}\text { Microtubule-associated protein } 1 \mathrm{~A} O S=\text { Rattus norvegicus } \mathrm{OX}=10,116 \\
\mathrm{GN}=\text { Map1a } \mathrm{PE}=1 \mathrm{SV}=1\end{array}$ & 32 & 299.3 & 89.0 & 111.1 & 0.00030 & 1.25 \\
\hline A0A0G2K728 & Cadherin-4 (Cdh4) & 3 & 94.4 & 89.0 & 111.1 & 0.035 & 1.25 \\
\hline P49805 & Regulator of G-protein signaling 9 (Rgs9) & 2 & 77.1 & 88.9 & 111.1 & 0.041 & 1.25 \\
\hline P22057 & Prostaglandin-H2 D-isomerase (Ptgds) & 9 & 21.3 & 88.8 & 111.2 & 0.011 & 1.25 \\
\hline Q8R490 & Cadherin 13 (Cdh13) & 13 & 78 & 88.7 & 111.3 & 0.00010 & 1.25 \\
\hline A0A0G2K613 & Uncharacterized protein & 22 & 19.8 & 88.7 & 111.3 & $<0.0001$ & 1.25 \\
\hline F1M7Z2 & IQ and AAA domain-containing protein 1 (lqca) & 1 & 99.3 & 88.5 & 111.6 & 0.0043 & 1.26 \\
\hline Q7TSE9 & HCLS1-associated protein X-1 (Hax1) & 9 & 31.4 & 88.3 & 111.8 & 0.0070 & 1.27 \\
\hline Q6J410 & Protein phosphatase 1 regulatory subunit 1B (Ppp1r1b) & 32 & 22.9 & 88.3 & 111.7 & 0.0016 & 1.27 \\
\hline P19944 & 605 acidic ribosomal protein P1 (Rplp1) & 57 & 11.5 & 87.9 & 112.1 & 0.047 & 1.28 \\
\hline AOAOG2KAY3 & Kininogen-1 (Kng1) & 12 & 44.2 & 87.4 & 112.6 & 0.00020 & 1.29 \\
\hline AOA0G2JSR6 & Guanine nucleotide-binding protein subunit gamma (Gng7) & 54 & 7.7 & 87.3 & 112.7 & 0.00080 & 1.29 \\
\hline P61459 & Pterin-4-alpha-carbinolamine dehydratase (Pcbd1) & 7 & 12 & 87.2 & 112.8 & 0.0020 & 1.29 \\
\hline B4F7E8 & Niban-like protein 1 (Fam129b) & 3 & 84.7 & 87.0 & 113.1 & 0.00050 & 1.30 \\
\hline F1M5V2 & GLI pathogenesis-related 2 (Glipr2) & 8 & 19 & 86.9 & 113.2 & 0.025 & 1.30 \\
\hline P19939 & Apolipoprotein C-I (Apoc1) & 10 & 9.9 & 86.8 & 113.2 & 0.011 & 1.30 \\
\hline Q5EBC0 & Inter alpha-trypsin inhibitor, heavy chain 4 (Itih4) & 9 & 103.7 & 86.7 & 113.3 & $<0.0001$ & 1.31 \\
\hline G3V8T7 & RCG51933, isoform CRA_a (Tdrkh) & 4 & 62 & 86.3 & 113.8 & 0.031 & 1.32 \\
\hline P47727 & Carbonyl reductase [NADPH] 1 (Cbr1) & 39 & 30.6 & 86.1 & 113.9 & 0.0052 & 1.32 \\
\hline A0A0G2JVX7 & Apolipoprotein A-IV (Apoa4) & 23 & 44.3 & 85.8 & 114.2 & 0.00020 & 1.33 \\
\hline P04094 & Proenkephalin-A (Penk) & 12 & 30.9 & 85.7 & 114.3 & 0.0010 & 1.33 \\
\hline A0A0G2JZB6 & Uncharacterized protein & 12 & 15.8 & 85.6 & 114.4 & 0.029 & 1.34 \\
\hline P02764 & Alpha-1-acid glycoprotein (Orm1) & 11 & 23.6 & 85.4 & 114.6 & $<0.0001$ & 1.34 \\
\hline P62749 & Hippocalcin-like protein 1 (Hpcal1) & 26 & 22.3 & 85.4 & 114.6 & 0.00080 & 1.34 \\
\hline P04177 & Tyrosine 3-monooxygenase (Th) & 28 & 55.9 & 85.3 & 114.7 & $<0.0001$ & 1.34 \\
\hline D3ZTN0 & Protein kinase, DNA-activated, catalytic polypeptide (Prkdc) & 0 & 471.8 & 85.2 & 114.8 & 0.010 & 1.35 \\
\hline Q00495 & Macrophage colony-stimulating factor 1 receptor (Csf1r) & 1 & 109.2 & 85.2 & 114.8 & 0.031 & 1.35 \\
\hline G3V7X2 & Scg2 protein (Scg2) & 4 & 66.6 & 85.1 & 114.9 & 0.0023 & 1.35 \\
\hline Q9QYU4 & Ketimine reductase mu-crystallin (Crym) & 39 & 33.5 & 85.0 & 115.1 & $<0.0001$ & 1.35 \\
\hline P07150 & Annexin A1 (Anxa1) & 17 & 38.8 & 84.9 & 115.1 & 0.0034 & 1.35 \\
\hline AOA0G2JZV3 & ATP/GTP-binding protein 1 (Agtpbp1) & 3 & 136.5 & 84.8 & 115.2 & 0.0061 & 1.36 \\
\hline Q68FY4 & Group specific component (Gc) & 28 & 53.5 & 84.8 & 115.2 & $<0.0001$ & 1.36 \\
\hline Q64240 & Protein AMBP (Ambp) & 8 & 38.8 & 84.8 & 115.2 & 0.00020 & 1.36 \\
\hline A0A0G2K3G0 & Histidine-rich glycoprotein (Hrg) & 4 & 60.3 & 84.7 & 115.3 & 0.0031 & 1.36 \\
\hline P08934 & Kininogen-1 (Kng1) & 5 & 70.9 & 84.6 & 115.4 & 0.028 & 1.37 \\
\hline Q9QX79 & Fetuin-B (Fetub) & 15 & 41.5 & 84.4 & 115.6 & 0.00010 & 1.37 \\
\hline F1M609 & Acyl-coenzyme A oxidase (Acox1) & 3 & 66 & 84.3 & 115.7 & 0.0086 & 1.37 \\
\hline A0A0G2K916 & Ceruloplasmin (Cp) & 10 & 123.6 & 83.8 & 116.2 & $<0.0001$ & 1.39 \\
\hline D3ZY96 & Neutrophilic granule protein (Ngp) & 20 & 19.4 & 83.8 & 116.2 & 0.0052 & 1.39 \\
\hline Q07936 & Annexin A2 (Anxa2) & 12 & 38.7 & 83.8 & 116.2 & $<0.0001$ & 1.39 \\
\hline D3ZGF1 & CD44 antigen (Cd44) & 7 & 52.8 & 83.7 & 116.3 & 0.0001 & 1.39 \\
\hline G3V6X7 & ProSAAS (LOC108348172) & 13 & 27.5 & 83.7 & 116.4 & 0.0021 & 1.39 \\
\hline MOR3N4 & Vesicle amine transport 1-like (Vat11) & 22 & 41.6 & 83.6 & 116.4 & $<0.0001$ & 1.39 \\
\hline Q5M878 & Serum amyloid A protein (Saa4) & 15 & 15 & 83.5 & 116.5 & 0.00010 & 1.39 \\
\hline Q5M818 & 39 S ribosomal protein L16, mitochondrial (Mrpl16) & 6 & 28.9 & 83.4 & 116.6 & 0.031 & 1.40 \\
\hline AOA0G2JWX4 & Keratin, type II cytoskeletal 2 epidermal (Krt2) & 6 & 69.1 & 83.4 & 116.6 & $<0.0001$ & 1.40 \\
\hline Q63433 & Serine/threonine-protein kinase N1 (Pkn1) & 1 & 104.4 & 83.2 & 116.8 & 0.0027 & 1.40 \\
\hline
\end{tabular}


Table 1 (continued)

\begin{tabular}{|c|c|c|c|c|c|c|c|}
\hline \multirow[t]{2}{*}{ Accession } & \multirow[t]{2}{*}{ Description } & \multirow[t]{2}{*}{ Coverage (\%) } & \multirow[t]{2}{*}{ MW (kDa) } & \multicolumn{2}{|c|}{ Average } & \multirow[t]{2}{*}{ p-value } & \multirow[t]{2}{*}{ FC } \\
\hline & & & & Sham & TBI & & \\
\hline AOA0G2K526 & Guanine nucleotide-binding protein G(olf) subunit alpha (Gnal) & 9 & 51.3 & 83.0 & 117.0 & 0.00010 & 1.41 \\
\hline P97738 & Neuronal pentraxin-2 (Nptx2) & 18 & 47.4 & 82.8 & 117.2 & 0.00010 & 1.42 \\
\hline P20761 & Ig gamma-2B chain $\mathrm{C}$ region (lgh-1a) & 7 & 36.5 & 82.2 & 117.8 & $<0.0001$ & 1.43 \\
\hline AOAOG2JYKO & Serine protease inhibitor A3N (LOC299282) & 27 & 67.9 & 81.8 & 118.2 & $<0.0001$ & 1.44 \\
\hline Q63910 & Alpha globin (Hba-a3) & 15 & 15.5 & 81.8 & 118.3 & 0.0056 & 1.45 \\
\hline P47728 & Calretinin (Calb2) & 15 & 31.4 & 81.2 & 118.9 & $<0.0001$ & 1.46 \\
\hline P01355 & Cholecystokinin (Cck) & 9 & 12.8 & 80.9 & 119.1 & 0.0029 & 1.47 \\
\hline D3ZQN7 & Laminin subunit beta 1 (Lamb1) & 1 & 197.3 & 80.8 & 119.2 & 0.038 & 1.48 \\
\hline D4AA52 & Murinoglobulin-1 (LOC297568) & 13 & 163.6 & 80.7 & 119.3 & $<0.0001$ & 1.48 \\
\hline P01048 & T-kininogen 1 (Map1) & 6 & 47.7 & 80.7 & 119.3 & 0.012 & 1.48 \\
\hline Q4FZU2 & Keratin, type II cytoskeletal 6A (Krt6a) & 7 & 59.2 & 80.4 & 119.7 & 0.017 & 1.49 \\
\hline P06866 & Haptoglobin (Hp) & 19 & 38.5 & 80.3 & 119.7 & $<0.0001$ & 1.49 \\
\hline Q510M1 & Apolipoprotein H (Apoh) & 11 & 38.4 & 80.3 & 119.8 & 0.011 & 1.49 \\
\hline Q6IFW6 & Keratin, type I cytoskeletal 10 (Krt10) & 13 & 56.5 & 80.2 & 119.8 & $<0.0001$ & 1.49 \\
\hline A0A0G2QC06 & Serotransferrin (Tf) & 24 & 107.3 & 80.1 & 120.0 & $<0.0001$ & 1.50 \\
\hline P02767 & Transthyretin (Ttr) & 20 & 15.7 & 79.8 & 120.2 & 0.00010 & 1.51 \\
\hline F7FHF3 & Serpin family F member 2 (Serpinf2) & 5 & 62.3 & 79.6 & 120.5 & 0.00010 & 1.51 \\
\hline D3ZFH5 & Inter-alpha-trypsin inhibitor heavy chain 2 (Itih2) & 4 & 92.3 & 79.4 & 120.6 & $<0.0001$ & 1.52 \\
\hline Q68G38 & Torsin-1A (Tor1a) & 3 & 37.9 & 79.3 & 120.7 & 0.034 & 1.52 \\
\hline A0A0G2JUX5 & Transcriptional activator protein Pur-beta (Purb) & 24 & 33.5 & 79.3 & 120.7 & $<0.0001$ & 1.52 \\
\hline P20156 & Neurosecretory protein VGF (Vgf) & 21 & 68.1 & 78.7 & 121.3 & 0.026 & 1.54 \\
\hline MORBF1 & Complement C3 (C3) & 21 & 186.2 & 78.6 & 121.5 & $<0.0001$ & 1.55 \\
\hline P00697 & Lysozyme C-1 (Lyz1) & 16 & 16.7 & 77.4 & 122.6 & 0.00040 & 1.58 \\
\hline A0A0G2JZ73 & Alpha-1-antiproteinase (Serpina1) & 39 & 46.6 & 77.4 & 122.6 & $<0.0001$ & 1.59 \\
\hline Q62930 & Complement component C9 (C9) & 2 & 62.2 & 77.3 & 122.8 & 0.00010 & 1.59 \\
\hline Q6P6Q2 & Keratin, type II cytoskeletal 5 (Krt5) & 15 & 61.8 & 77.1 & 122.9 & $<0.0001$ & 1.59 \\
\hline G3V9R9 & Afamin (Afm) & 15 & 69.2 & 76.9 & 123.2 & $<0.0001$ & 1.60 \\
\hline P24090 & Alpha-2-HS-glycoprotein (Ahsg) & 26 & 38 & 76.6 & 123.4 & $<0.0001$ & 1.61 \\
\hline P20059 & Hemopexin (Hpx) & 23 & 51.3 & 76.5 & 123.6 & $<0.0001$ & 1.62 \\
\hline A0A0G2JSK1 & RCG20603 (Serpina3c) & 28 & 46.4 & 76.3 & 123.7 & $<0.0001$ & 1.62 \\
\hline $\mathrm{AOAOH} 2 \mathrm{UHJ} 1$ & Protein S100-A9 (S100a9) & 11 & 15.2 & 76.0 & 124.0 & $<0.0001$ & 1.63 \\
\hline Q6IMF3 & Keratin, type II cytoskeletal 1 (Krt1) & 8 & 64.8 & 75.7 & 124.3 & $<0.0001$ & 1.64 \\
\hline F7F8G2 & Bromodomain testis-specific protein (Brdt) & 1 & 106.7 & 75.6 & 124.4 & $<0.0001$ & 1.65 \\
\hline P12346 & Serotransferrin (Tf) & 31 & 76.3 & 75.3 & 124.7 & 0.00010 & 1.66 \\
\hline D3ZJF8 & Fc fragment of lgG-binding protein (Fcgbp) & 0 & 275 & 75.2 & 124.9 & $<0.0001$ & 1.66 \\
\hline $\mathrm{AOAOH} 2 \mathrm{UH} 15$ & Serine protease inhibitor A3N (Serpina3n) & 24 & 45.5 & 74.4 & 125.6 & $<0.0001$ & 1.69 \\
\hline Q63041 & Alpha-1-macroglobulin (A1m) & 10 & 167 & 74.4 & 125.7 & $<0.0001$ & 1.69 \\
\hline P10959 & Carboxylesterase 1C (Ces1c) & 11 & 60.1 & 74.2 & 125.9 & $<0.0001$ & 1.70 \\
\hline Q03626 & Murinoglobulin-1 (Mug1) & 13 & 165.2 & 73.7 & 126.3 & $<0.0001$ & 1.71 \\
\hline Q5BJY9 & Keratin, type I cytoskeletal 18 (Krt18) & 6 & 47.7 & 73.6 & 126.4 & 0.00040 & 1.72 \\
\hline $\mathrm{AOAOH} 2 \mathrm{UI} 36$ & Uncharacterized protein & 14 & 17 & 71.9 & 128.2 & $<0.0001$ & 1.78 \\
\hline P50115 & Protein S100-A8 (S100a8) & 22 & 10.2 & 71.6 & 128.4 & $<0.0001$ & 1.79 \\
\hline P42930 & Heat shock protein beta-1 (Hspb1) & 41 & 22.9 & 71.0 & 129.0 & $<0.0001$ & 1.82 \\
\hline Q6IFV1 & Keratin, type I cytoskeletal 14 (Krt14) & 15 & 52.7 & 70.8 & 129.2 & $<0.0001$ & 1.82 \\
\hline P04639 & Apolipoprotein A-I (Apoa1) & 41 & 30 & 70.8 & 129.2 & $<0.0001$ & 1.83 \\
\hline P02770 & Serum albumin (Alb) & 64 & 68.7 & 69.9 & 130.2 & $<0.0001$ & 1.86 \\
\hline Q62669 & Globin a1 (LOC103694855) & 46 & 16 & 68.8 & 131.2 & $<0.0001$ & 1.91 \\
\hline BOBNN3 & Carbonic anhydrase 1 (Ca1) & 8 & 28.3 & 67.7 & 132.3 & 0.00010 & 1.95 \\
\hline
\end{tabular}


Table 1 (continued)

\begin{tabular}{|c|c|c|c|c|c|c|c|}
\hline \multirow[t]{2}{*}{ Accession } & \multirow[t]{2}{*}{ Description } & \multirow[t]{2}{*}{ Coverage $(\%)$} & \multirow[t]{2}{*}{ MW (kDa) } & \multicolumn{2}{|c|}{ Average } & \multirow[t]{2}{*}{ p-value } & \multirow[t]{2}{*}{ FC } \\
\hline & & & & Sham & TBI & & \\
\hline MORCXO & Pterin-4 alpha-carbinolamine dehydratase 2 (Pcbd2) & 22 & 14.1 & 66.4 & 133.6 & 0.039 & 2.01 \\
\hline P02091 & Hemoglobin subunit beta-1 (Hbb) & 82 & 16 & 64.3 & 135.7 & $<0.0001$ & 2.11 \\
\hline Q6IFU7 & Keratin, type I cytoskeletal 42 (Krt42) & 13 & 50.2 & 62.0 & 138.0 & $<0.0001$ & 2.22 \\
\hline P11517 & Hemoglobin subunit beta-2 (Hbb2) & 78 & 16 & 59.3 & 140.8 & $<0.0001$ & 2.38 \\
\hline P01946 & Hemoglobin subunit alpha-1/2 (Hba1) & 63 & 15.3 & 54.9 & 145.1 & $<0.0001$ & 2.64 \\
\hline A0A0G2JSH5 & Serum albumin (Alb) & 65 & 68.7 & 47.6 & 152.4 & 0.00010 & 3.21 \\
\hline F1LRV4 & Heat shock 70 kDa protein 4 (Hspa4) & 41 & 94 & 20.9 & 179.1 & $<0.0001$ & 8.56 \\
\hline
\end{tabular}

$M W$ molecular weight, $T B /$ traumatic brain injury, FC fold change

alpha-carbinolamine dehydratase), Pcbd2, Calb2 (calbindin 2), Penk (proenkephalin), and Hbb. Hspb1 (heat shock protein b1) interacted with Hspa4, Anxa1 (annexin A1), and Cck (cholecystokinin).

\section{Validation of differentially expressed proteins by PRM}

A PRM assay was developed to verify the abundance alterations of 23 upregulated proteins formerly identified by quantitative iTRAQ analysis (Fig. 1). As illustrated in Table 2, among the 23 proteins, seven were inconsistent with previous outcomes yielded by quantitative analysis. Among these, F1LRV4 (Hspa4) and Q6IFU7 (Krt42) were not significantly changed, while Q6IFV1, Q6IMF3, Q6P6Q2, D3ZJF8 and G3V9R9 (Krt14, Krt1, Krt5, Fcgbp and Afamin) were downregulated. The other 16 proteins were consistent with previous iTRAQ analysis outcomes, 11 of which were displayed in Fig. 4a. Additional file 2: Table S2 showed detailed quantitation of all peptides and proteins. The intensities and peak areas of two peptides in TTR were shown in Fig. 4b. The accurate intensities and peak areas of two peptides in each sample were shown in Additional file 3: Figure S1 and Additional file 4: Figure S2. The intensity of TTR ascended significantly after CCI, in agreement with the iTRAQ analysis.

\section{Histopathology after T4 treatment}

To evaluate the effects of T4 on alleviation of TBIinduced cerebrovascular histopathology, brain water content and BBB integrity were examined (Fig. 1). First, brain lesion volumes in the four groups (sham + saline, sham $+\mathrm{T} 4$, TBI + saline, and TBI $+\mathrm{T} 4$ ) were examined, and it was found that T4 significantly reduced lesion size in TBI rats, as reflected in brain sections shown in Fig. 5a. Lesion volume in the TBI+saline group was significantly higher compared with the sham + saline group ( $1.90 \pm 0.21$ vs. $\left.13.20 \pm 2.34 \mathrm{~mm}^{3}, p=0.0011\right)$. When comparing lesion volume in the $\mathrm{TBI}+\mathrm{T} 4$ group, the difference was also significant $(13.20 \pm 2.34$ vs. $6.12 \pm 1.69, p=0.013)$.

Brain water content was detected using a wet-todry ratio. According to Fig. 5b, there was no change in water content between two sham groups (exposed or not exposed to T4 $[78.03 \pm 0.26 \%$ vs. $78.78 \pm 0.67 \%$, $p=0.14]$ ), while water content in the ipsilateral hemisphere significantly increased after CCI without exposure to T4 $(78.03 \pm 0.26 \%$ vs. $80.90 \pm 0.37 \%, p=0.0085)$. After CCI rats were exposed to T4, the water content significantly decreased $(79.46 \pm 0.25 \%, p=0.0049)$, but it was still significantly higher than the sham + saline group $(p=0.0024)$. This indicated that the addition of T4 may improve brain edema. The extravasation of EB dye was examined and is shown in Fig. 5c. After $24 \mathrm{~h}$ post-CCI, the extravasation significantly increased in the TBI+ saline group $(76.16 \pm 10.19 \mu \mathrm{g} / \mathrm{g}$ vs. $164.30 \pm 9.44 \mu \mathrm{g} / \mathrm{g}, p=0.0004)$. Extravasation of the $\mathrm{TBI}+\mathrm{T} 4$ group was also significantly lower than the $\mathrm{TBI}+$ saline group $(110.30 \pm 13.62 \mu \mathrm{g} / \mathrm{g}, \quad p=0.0049)$. However, it was also higher than the sham + saline group $(p=0.025)$. This indicated improved BBB integrity after exposure to T4. Brain samples also confirmed these results. In general, the aforementioned outcomes indicated that T4 alleviated TBI-induced brain histopathology, including BBB impairment and cerebral edema.

\section{Behavioral outcomes}

To examine the impacts of $\mathrm{T} 4$ on improving behavioral performance and neurological function of rats postCCI, mNSS and MWM tests were conducted (Fig. 1). As shown in Fig. 6a, on days 1, 3, 5, and 7 post-CCI, mNSS of the $\mathrm{TBI}+$ saline group was significantly higher than the sham + saline groups ( $p<0.0001$ in all groups). On days 1 and 3, mNSS of the TBI + T4 group was also significantly higher than the sham + saline group $(p=0.0002$ on day 1 and $p=0.026$ on day 3 ). However, on day 5 , the difference between the two groups was not significant $(p=0.14)$. 
(See figure on next page.)

Fig. 3 Bioinformatic analysis for differentially expressed proteins in the TBI and sham rats. a Biological process (BP). b cell components (CC). c molecular function (MF). Darker colors indicate higher statistical significancy. $\mathbf{d}$ Bubble chart shows KEGG analysis of differential genes. The horizontal axis represents rich factor (ratio of the sum of differential genes enriched in a pathway to the number of genes annotated by the pathway). Bubble size indicates the number of proteins included in each pathway, and different colors indicate different $p$-values. e Protein-protein interaction (red = upregulated protein; green = downregulated protein). Rounded rectangles represent KEGG pathways. Lines show interactions between multiple proteins or between proteins and pathways

Additionally, significant differences were observed between the TBI + saline and TBI + T4 groups on all four days ( $p=0.011$ on day $1, p<0.01$ on days 3,5 , and 7 ).

The MWM test was performed at the beginning of day 7 (Fig. 6b). On day 7, differeces of escape latency among the four groups were not significant. After day 8 , escape latency of the TBI + T4 group dropped gradually, and the TBI + saline group maintained a level higher than the sham + saline group $(p=0.0020$ on day 8 , $p=0.0090$ on day $9, p=0.0120$ on day 10 , and $p<0.0001$ on day 11). After day 10, a significant difference between the TBI + saline and TBI $+\mathrm{T} 4$ groups was detected $(p=0.0098$ on day 10 and $p=0.030$ on day 11$)$. On test day (day 12), escape latency of the TBI+ saline group significantly increased compared to the sham + saline group (92.00 \pm 36.39 vs. $16.18 \pm 13.46, p=0.0007)$. After treatment with $\mathrm{T} 4$, escape latency dropped dramatically $(35.30 \pm 21.85, p=0.0084)$.

On day 12 post-CCI, the number of times rats crossed the location where the platform was previously placed was tested. As shown in Fig. 6d, rats in the TBI+saline group crossed the location less frequently than the sham + saline group $(0.67 \pm 0.82$ vs. $2.00 \pm 0.63 p=0.010)$, and the TBI $+\mathrm{T} 4$ group crossed more frequently than the $\mathrm{TBI}+$ saline group $(1.667 \pm 0.82 p=0.060)$. Time spent in the platform quadrant on test day was also measured (Fig. 6e), and the TBI+ saline group spent significantly less time in the quadrant than the sham + saline group $(21.15 \pm 6.16$ vs. $34.65 \pm 11.15, p=0.027)$ and TBI $+\mathrm{T} 4$ group (33.42 $\pm 3.706, p=0.0019)$. Furthermore, difference in escape latency between the TBI+saline and sham + saline groups on test day was also significant $(p=0.0007)$. The effects of T4 on behavioral outcome improvements were evident.

\section{Proteomic analysis after T4 treatment}

We also conducted iTRAQ analysis of cortices after CCI and T4 treatment. Through whole proteomic profiling, a series of proteins and pathways potentially involved in T4 effects were identified. A total of 199 proteins were differentially expressed comparing sham + saline and $\mathrm{TBI}+$ saline groups and 51 differentially expressed proteins were identified comparing $\mathrm{TBI}+$ saline and $\mathrm{TBI}+\mathrm{T} 4$ groups. Among these differential proteins, 23 proteins were altered by TBI and reversed by T4 treatment (Additional file 1: Table S1; Fig. 7a, b). Some proteins such as Lgals3, Hspb1, Vim, Gfap, Fabp7, Pltp, Msn, Ctsb, and Apoe were upregulated after TBI and reversed by $\mathrm{T} 4$ treatment, and the expression trend of proteins like Abca1, Clpp, Mtco1, Nefm and Nefl were significantly changed by T4 treatment (Fig. 7c). Most reversed proteins were upregulated after TBI and downregulated by T4 (Fig. 7c). The 51 proteins altered by $\mathrm{T} 4$ treatment were enriched for GO terms. Top $10 \mathrm{GO}$ terms and genes associated with these terms were shown in Fig. 7d. Differential proteins were mostly associated with intermediate filament organization, cholesterol transportation and axonal regeneration.

\section{Discussion}

Quantitative proteomics has been widely applied to the detection of differential proteins and pathways in many disease models, including TBI. In this study, an 8-plex iTRAQ analysis enabled the investigation of large-scale protein alterations. In total, 3937 proteins were identified, and 146 proteins were found to be differentially expressed. Some of these proteins have been discovered to be related to TBI. cAMP signaling pathway-related proteins, guanine nucleotide-binding protein $\mathrm{G}$ (Gnal) and phosphodiesterase 10A (Pde10a), were upregulated significantly in this study. KEGG also showed that the cAMP signaling pathway was significant. This was in agreement with a previous study by Song et al. [45]. This study further confirmd the relationship between cAMPsignaling pathway and TBI.

Recently, many studies have proven that Hsp70 increased rapidly in traumatically injured brains of mammals, including humans, and has protective effects on brain rehabilitation [54, 55]. This phenomenon was also reflected in the proteomic outcomes; although, no significant increase was detected in PRM. In addition, Hspb1 was upregulated in both iTRAQ and PRM, which has been associated with multiple sclerosis in the spinal cord [56]. Many proteins in the heat shock protein family have been found to be protective in neurodegenerative diseases and brain trauma $[57,58]$; thus, further research is required to explore the effects of other proteins in this family on posttraumatic protection. Inconsistency between PRM and iTRAQ also exists. For example, Hsp70 upregulated eight folds in iTRAQ but did not significantly change in PRM. In 


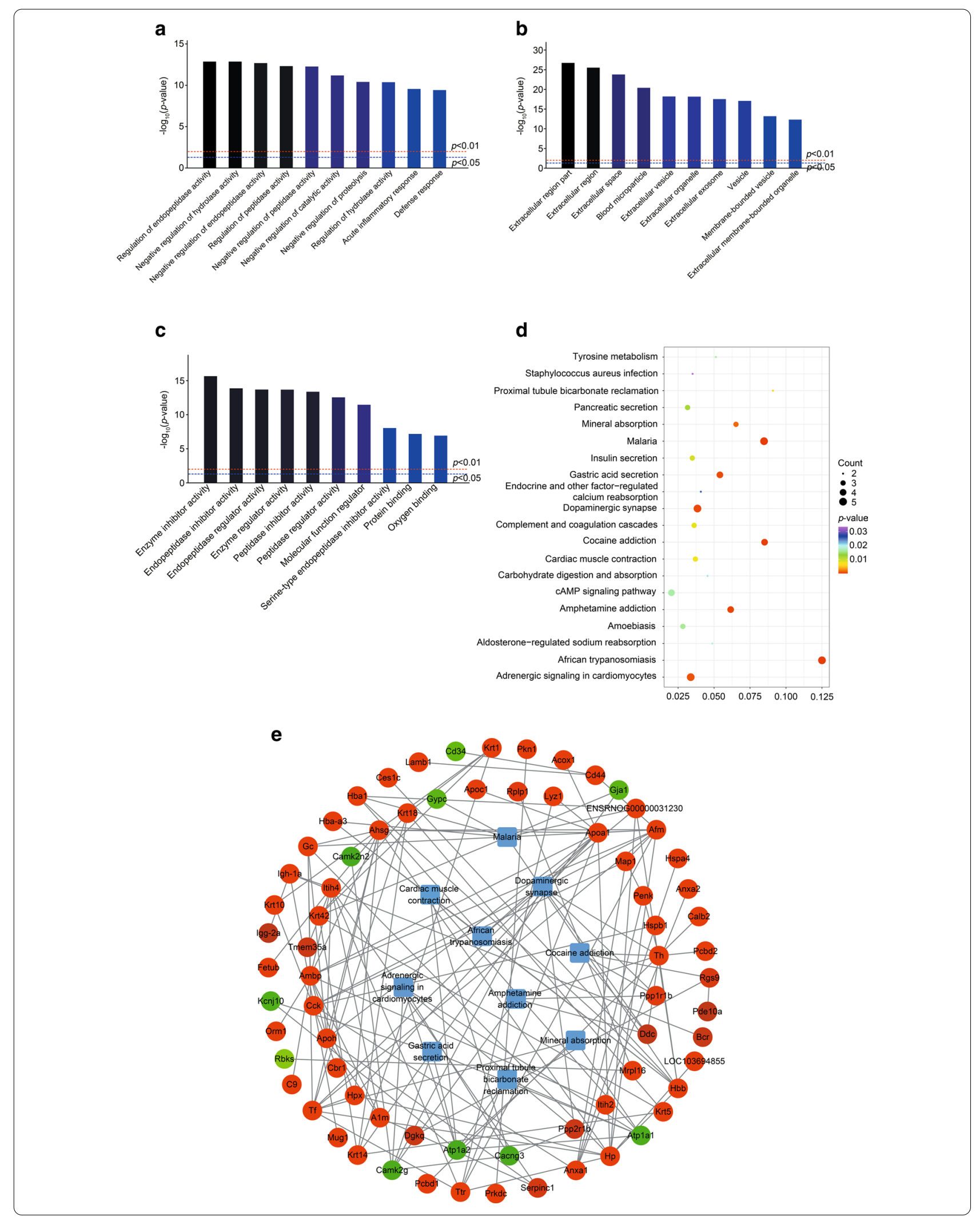


Table 2 Verification of differentially expressed proteins after TBI by PRM

\begin{tabular}{|c|c|c|c|c|c|}
\hline \multirow[t]{2}{*}{ Accession } & \multirow[t]{2}{*}{ Gene name } & \multicolumn{2}{|c|}{ Average total normalized area } & \multirow[t]{2}{*}{ Fold change } & \multirow[t]{2}{*}{ p-value } \\
\hline & & Sham & TBI & & \\
\hline A0A0G2JSK1 & Serpina3c & $587,193.8154$ & $967,199.9746$ & 1.65 & 0.0010 \\
\hline A0A0G2JZ73 & Serpina1 & $358,594,583.9$ & $661,025,493$ & 1.84 & $<0.0001$ \\
\hline $\mathrm{AOAOH} 2 \mathrm{UH} 15$ & Serpina3n & $15,925,972.06$ & $30,105,445.75$ & 1.89 & $<0.0001$ \\
\hline D3ZJF8 & Fcgbp & $492,977,070$ & $364,252,286$ & 0.74 & 0.00020 \\
\hline F1LRV4 & Hspa4 & $315,888,493.8$ & $319,391,993$ & 1.01 & 0.48 \\
\hline G3V9R9 & Afm & $9,171,981.438$ & $6,262,567.344$ & 0.68 & 0.058 \\
\hline MORBF1 & C3 & $33,341,743.73$ & $88,878,077.44$ & 2.66 & $<0.0001$ \\
\hline P01946 & Hbal & $859,325,340.5$ & $3,225,272,654$ & 3.75 & $<0.0001$ \\
\hline P02091 & $\mathrm{Hbb}$ & $2,007,838,409$ & $6,847,982,715$ & 3.41 & $<0.0001$ \\
\hline P02767 & Ttr & $152,664,429.5$ & $274,579,978.7$ & 1.80 & $<0.0001$ \\
\hline P02770 & Alb & $1,334,438,359$ & $1,462,217,836$ & 1.10 & 0.099 \\
\hline P11517 & $\mathrm{Hbb} 2$ & $1,987,823,049$ & $6,870,701,039$ & 3.46 & $<0.0001$ \\
\hline P20059 & Hpx & $81,400,524.38$ & $143,040,114.8$ & 1.76 & $<0.0001$ \\
\hline P20156 & Vgf & $17,525,134.63$ & $28,274,163.63$ & 1.61 & $<0.0001$ \\
\hline P24090 & Ahsg & $11,552,915.97$ & $25,108,376.28$ & 2.17 & $<0.0001$ \\
\hline P42930 & Hspb1 & $5,132,833.438$ & $18,194,532.5$ & 3.54 & $<0.0001$ \\
\hline Q03626 & Mug1 & $70,921,905.59$ & $149,480,671.3$ & 2.11 & $<0.0001$ \\
\hline Q62669 & LOC103694855 & $487,524,419.4$ & $1,385,746,698$ & 2.84 & $<0.0001$ \\
\hline Q63041 & $\mathrm{A} 1 \mathrm{~m}$ & $9,261,727.438$ & $18,843,111.94$ & 2.03 & $<0.0001$ \\
\hline Q6IFU7 & Krt42 & $40,789,820$ & $35,523,088.63$ & 0.87 & 0.31 \\
\hline Q6IFV1 & Krt14 & $927,194,854$ & $778,350,430.6$ & 0.84 & 0.00030 \\
\hline Q6IMF3 & Krt1 & $1,326,149,813$ & $947,186,885.5$ & 0.71 & $<0.0001$ \\
\hline Q6P6Q2 & Krt5 & $623,317,271.3$ & $427,289,138.7$ & 0.69 & 0.0095 \\
\hline
\end{tabular}

$T B I$ traumatic brain injury; $P R M$ parallel reaction monitoring

proteomic analysis, fake positive is possible, because qualitative analysis of proteins is scored based on the matching degree and the number of peptides, the result is always a possibility. Also, Hsp70 has homologous proteins, which could affect the precision of identification. Thus, a verification like PRM becomes necessary. Upregulation of S100 calcium-binding protein A9 (S100A9) has been proposed to be related to acute neuroinflammation [54], which was consistent with the proteomic outcomes in this study. In addition, a recent study demonstrated that genetic deletion of S100A9 had a neuroprotective effect, which indicates that S100A9 may be a potential treatment target for brain trauma [59]. Also, Hpx protected BBB integrity in cerebral ischemia [60]. Gc is a binding protein and transports vitamin $\mathrm{D}$, and vitamin $\mathrm{D}$ levels are significantly related to the severity of head injury. Vitamin D replacement after trauma may be beneficial [61]. Penk is a protein found in the endogenous opioid system and may be useful for predicting clinical outcomes of severe TBI, and it has been used to relieve craniofacial pain in rat models of TBI $[62,63]$.

The relationship between cocaine addiction and TBI was reflected by the KEGG results. Some previous research has reached similar conclusions. Individuals with cocaine addiction were found to have a higher proportion of TBI history than healthy individuals [64]. TBI has been proposed to augment the rewarding effects of some psychostimulants and has been linked with increased vulnerability to substance abuse $[65,66]$. Drug addiction has been associated with dopamine, a neurotransmitter that controls motivation and reward [67]. This KEGG analysis also revealed that some upregulated proteins participated in dopaminergic synapse activity, and some proteins in this pathway (i.e., Camk2g, Ppp1r1b, Th, and Ddc) were also involved in cocaine or amphetamine addiction pathways. These results confirmed previous research findings that brain trauma may enhance drug addiction via dopamine synapse pathways. Interestingly, adrenergic signaling in cardiomyocytes and cardiac muscle contraction were related to TBI. Previous studies have illustrated that there were interactions between the heart and brain after TBI and that TBI may be a risk factor for myocardial dysfunction $[68,69]$.

Thyroid hormones have been proven essential for neuronal protection, recovery, and regeneration after brain trauma and are considered a potential therapeutic method 


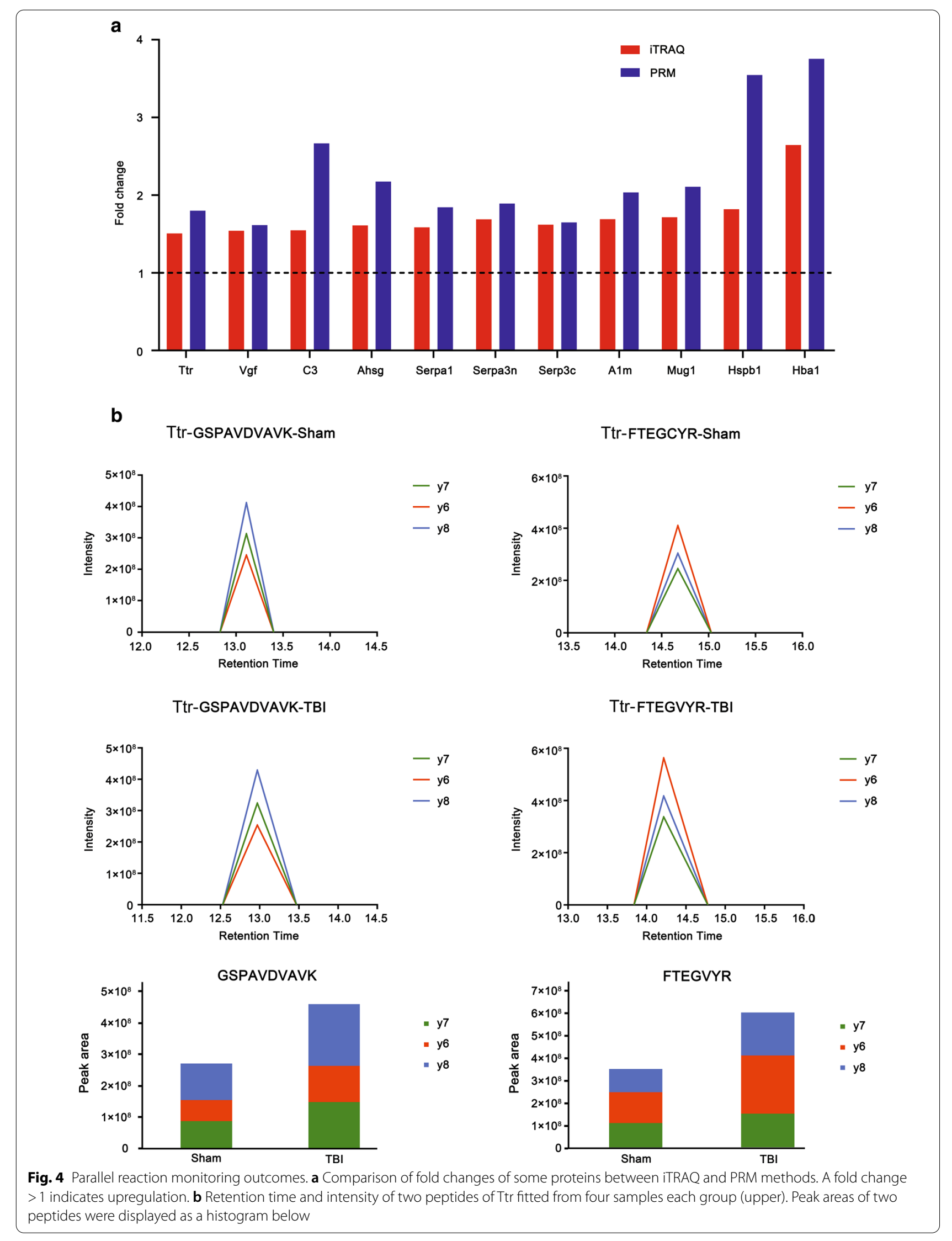




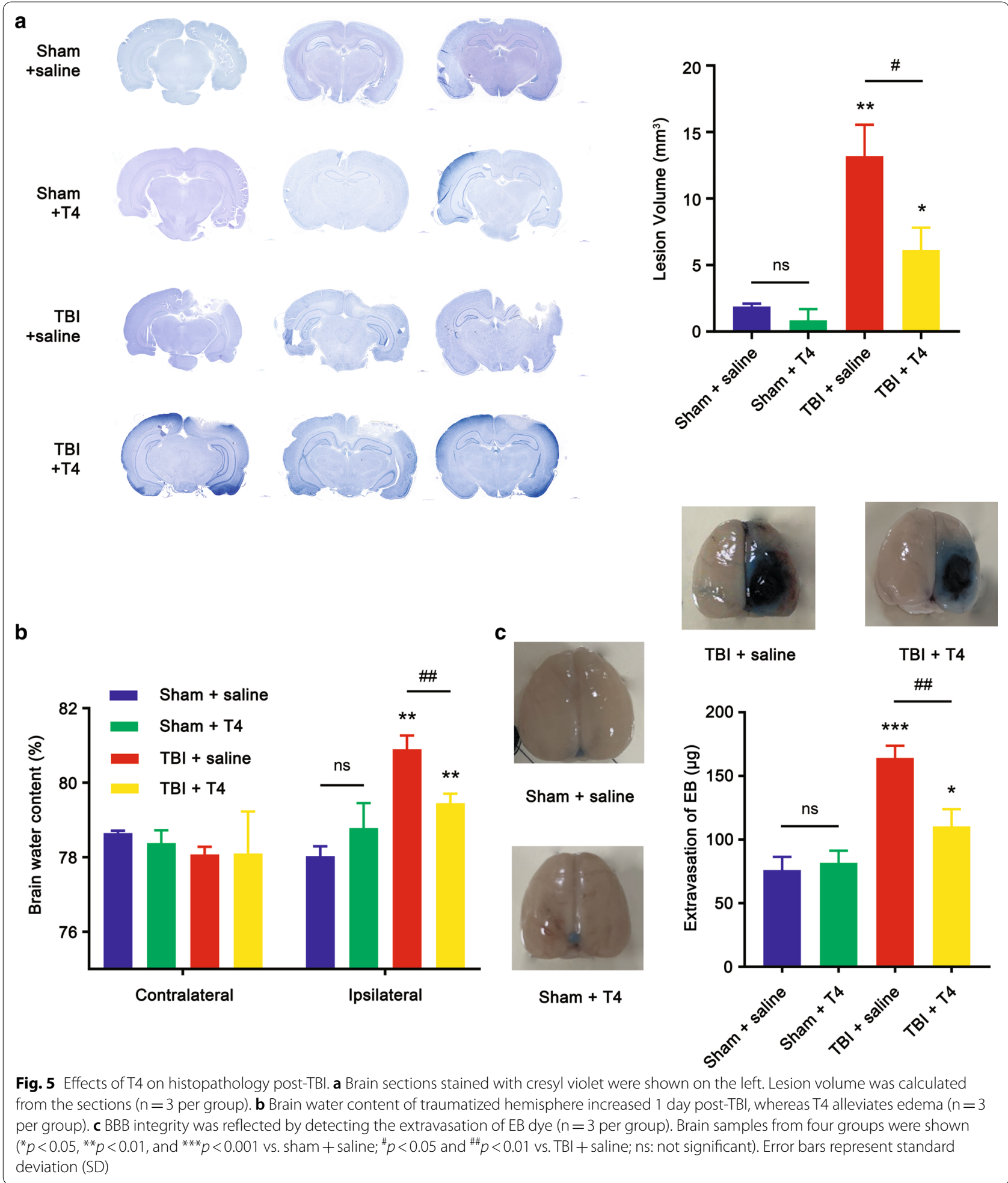

$[49,51,70]$. Ttr, a major carrier of thyroid hormones that can cross the BBB and preferentially bind T4 [71], was shown to be upregulated post-TBI by iTRAQ analysis and verified by PRM. Recently, using unbiased single-cell sequencing, Arneson et al. demonstrated that upregulation of Ttr in different cell types in hippocampus after TBI and the use of $\mathrm{T} 4$ to modulate Ttr may mitigate behavioral outcomes and genetic problems caused by TBI in mice. 

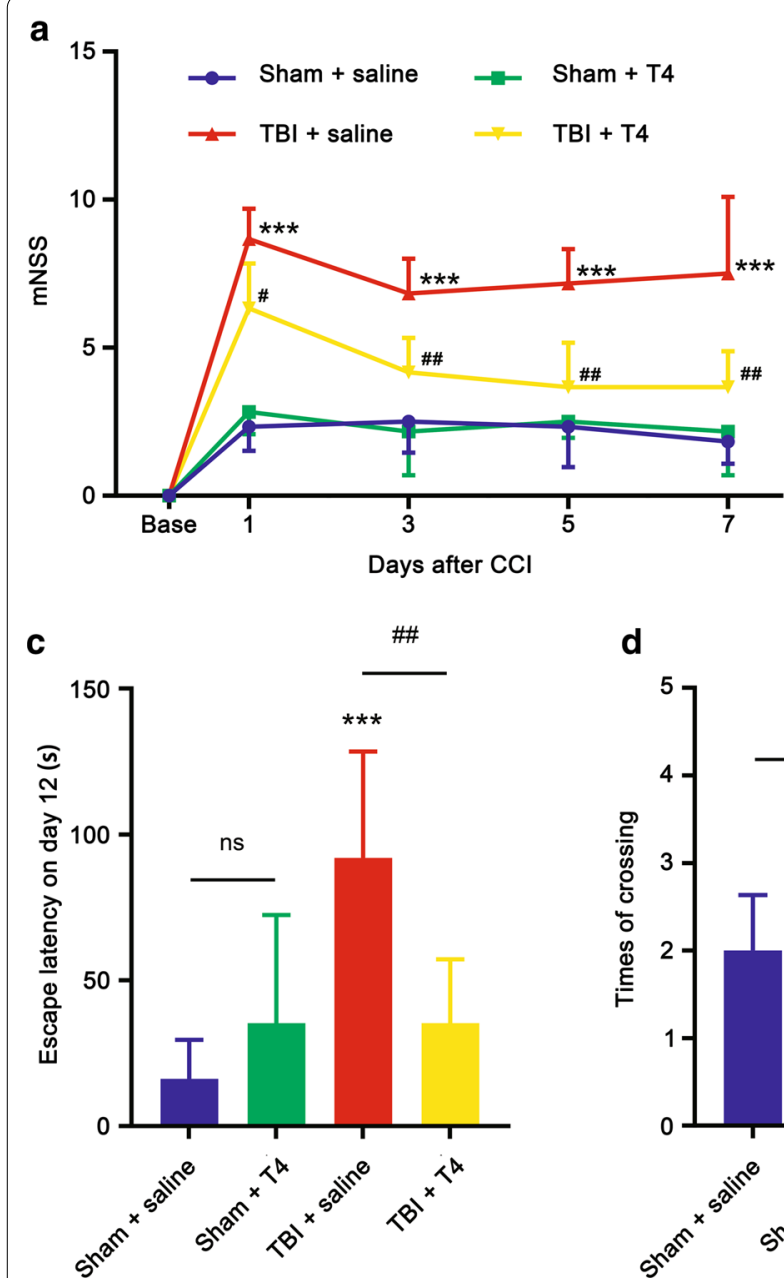

b

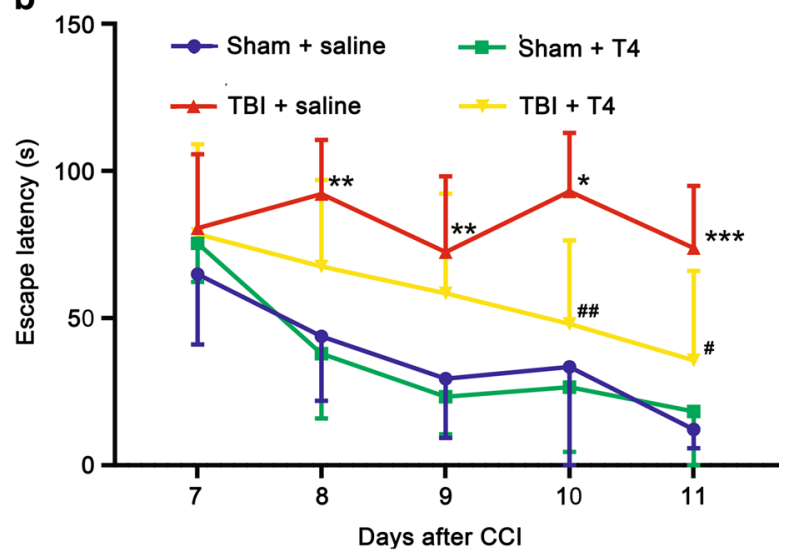

d e

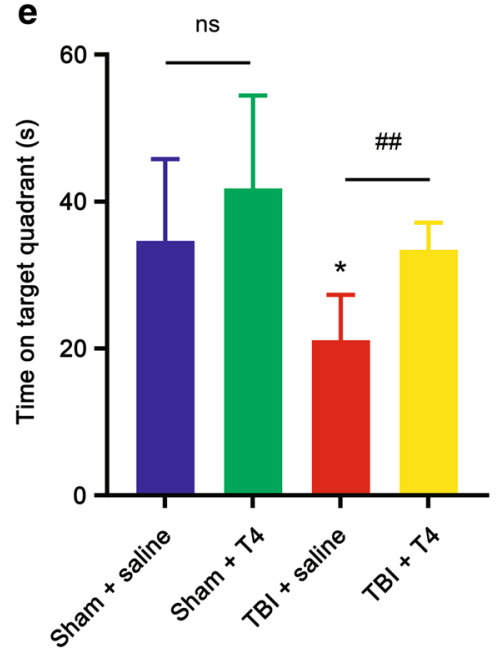

Fig. 6 Behavioral outcomes. a mNSS score on days 1, 3, 5, and 7 post-TBI ( $n=6$ per group). b Escape latency in MWM test 7 to 11 days post-TBI ( $n=6$ per group). On test day (day 12), c escape latency, $\mathbf{d}$ number of platform crossings, and e time spent in platform quadrant were detected ( $n=6$ per group; ${ }^{*} p<0.05,{ }^{* *} p<0.01$, and ${ }^{* * *} p<0.001$ vs. sham + saline; ${ }^{*} p<0.05$ and ${ }^{\# \#} p<0.01$ vs. TBI + saline; ns: not significant). Error bars represent $\mathrm{SD}$

The research concluded that upregulation of Ttr after TBI was due to a lack of thyroid hormones in the cerebrum and that T4 may reverse the deficiency. T4 treatment may reverse expression patterns of many $\mathrm{T} 4$ transporter genes, among which Ttr showed the most significant fold changes [72]. Our study confirmed that using T4 can ameliorate brain edema, improve integrity of the $\mathrm{BBB}$, and improve cognitive outcome in TBI-affected rats. Some of the effects originated from the physiological functions of thyroxine itself, while some may be achieved by regulating Ttr. Another study also confirmed that Ttr increased as the severity of TBI strengthened; thus, Ttr may be used as a criterion to assess the severity of TBI [43]. The important role of Ttr in TBI has been further confirmed by quantitative proteomics in this study.

Proteomic profile between TBI and TBI + T4 revealed pathways responsible for T4 treatment. A total of 51 proteins differentially expressed after T4 treatment and 23 of the proteins reversed the alteration after TBI. Among the 23 proteins, many of them have been studied and shown to be associated with neuroinflammation, brain edema and BBB integrity. For example, Galectin-3 (Lgals3) is important in brain inflammatory response in neurodegenerative diseases $[73,74]$ and inhibiting galectin-3 has been proved to ameliorate brain edema in subarachnoid hemorrhage [75]. Hspb1 (Hsp27) responds to stress and has some neuroprotective effects. Activation of Hsp27 could ameliorate intracerebral hemorrhage-induced secondary brain injury and attenuate blood-brain barrier disruption [76, 77]. Vimentin and GFAP are intermediate filaments involved in neural plasticity and regeneration and play important roles in responses to stress such as injury, ischemia and neurodegeneration [78]. 
a Sham vs $\mathrm{TBI} \quad \mathrm{TBI}+\mathrm{T} 4$ vs $\mathrm{TBI}$

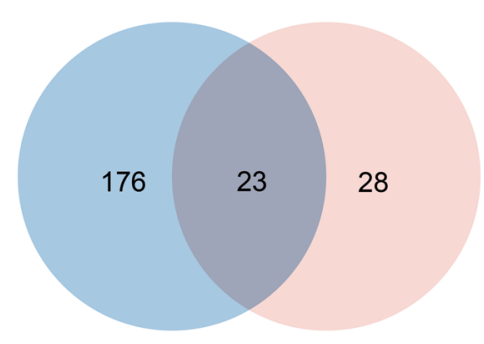

C

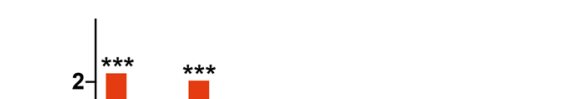

b

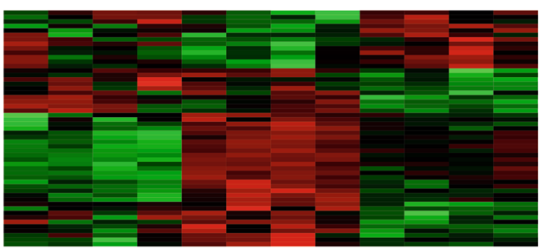

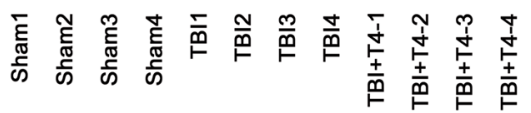

TBI vs Sham

- TBI+T4 vs TBI
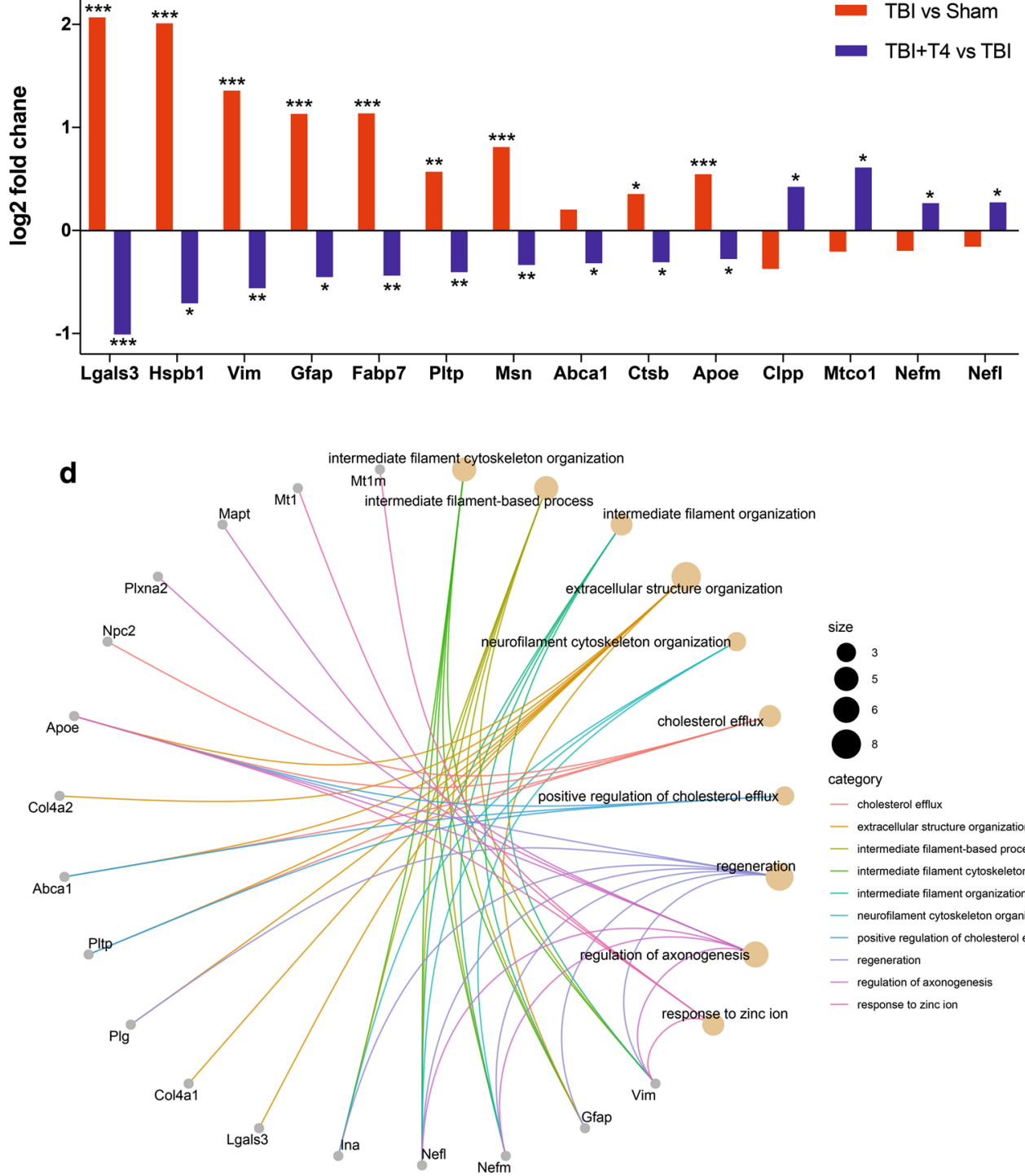

category

- cholesterol efflux

- extracellular structure organization

- intermediate filament-based process

- intermediate filament cytoskeleton organization

- intermediate filament organization

- neurofilament cytoskeleton organization

- positive regulation of cholesterol efflux

- regeneration

— regulation of axonogenesis

- response to zincion

Fig. 7 Proteomic analysis and GO enrichment after TBI and T4 treatment. a Venn diagram, TBI vs. sham and TBI + T4 vs. TBI show overlap in differentially expressed proteins. $\mathbf{b}$ Heatmap shows 51 proteins significantly changed after T4 treatment. The number in the color scale indicates $z$-score. c Examples of proteins significantly changed after T4 treatment. d Top $10 \mathrm{GO}$ terms and proteins associated with these terms. Size of the circles represents number of proteins associated with the terms. $\left(n=4\right.$ per group; ${ }^{*} p<0.05$, ${ }^{* *} p<0.01$, and ${ }^{* * *} p<0.001$ ) 
GFAP has also been confirmed to be a sensitive biomarker for TBI diagnosis and prediction, which was consistent to our proteomic outcome [79]. Fatty acidbinding protein-7 (Fabp7) in astrocytes was associated with glial differentiation, proliferation and neurogenesis, and has been confirmed to protect BBB integrity after TBI [80, 81]. Phospholipid transfer protein (PLTP) and moesin have been confirmed to be protective in BBB integrity by involving cerebrovascular remodeling and maintaining the function of transporters at $\mathrm{BBB}$ respectively [82, 83]. ATP-binding cassette transporter member A1 (ABCA1) and apolipoprotein E (ApoE) are major cholesterol transporters that play essential roles in cholesterol homeostasis in brain. ABCA1/ApoE were reported in many research about their effects in neural restoration after stroke [84]. Some of these upregulations after TBI could have protective effects and some were just reactive. With T4 treatment, possibly because the BBB integrity, vascular pathology and brain edema were alleviated, these upregulations were reversed. Some proteins downregulated after TBI but upregulated significantly with T4 treatment, although the downregulation after TBI was not significant in most proteins. Among these proteins, Nefm and Nefl were enriched in GO terms such as regeneration and axonogenesis, which probably indicated that $\mathrm{T} 4$ treatment contributes to neural regeneration. Cytochrome $c$ oxidase (Mtcol) is an enzyme in the mitochondrial electron transport chain for oxidative phosphorylation [85], upregulation with $\mathrm{T} 4$ treatment suggested mitochondrial function was restored. Combining with GO enrichment, T4 treatment could ameliorate brain inflammation, edema and BBB damage and involves in neural regeneration, cholesterol transporting, intermediate filament organization and mitochondrial function pathways.

Based on iTRAQ proteomic profiling, a data set to summarize protein alterations after TBI was generated. The PRM method validated some proteins, such as Ttr, as reliable biomarkers or treatment targets for TBI. Proteomic analysis also revealed pathways and differential proteins involved in T4 treatment after TBI. iTRAQ proteomic technologies can effectively facilitate the exploration of important differential proteins and help detect disease mechanisms.

\section{Supplementary Information}

The online version contains supplementary material available at https://doi. org/10.1186/s13041-021-00739-0.

Additional file 1: Table S1. Identified proteins and differentially expressed proteins in iTRAQ.
Additional file 2: Table S2. Quantitative data of peptides and proteins in PRM.

Additional file 3: Figure S1. Chromatograms of fragment ions and peak area of peptide FTEGVY corresponding to Ttr.

Additional file 4: Figure S2. Chromatograms of fragment ions and peak area of peptide GSPAVDVAVK corresponding to Ttr.

\section{Abbreviations}

iTRAQ: Isobaric tags for relative and absolute quantitation; LC-MS/MS: Liquid chromatography-tandem mass spectrometry; mNSS: Modified neurological severity scores; MWM: Morris water maze; PRM: Parallel reaction monitoring; RPLC: Reversed-phase liquid chromatography; T4: Thyroxine; TBI: Traumatic brain injury; Ttr: Transthyretin.

\section{Acknowledgements}

We thank LetPub (www.letpub.com) for its linguistic assistance during the preparation of this manuscript.

\section{Authors' contributions}

YZ and HM conceived of and designed the research plans. ZZ, YZ, HM, JY, PW, $\mathrm{RL}$, and RZ established the CCI model and isolated protein samples from cerebral cortex tissues. ZZ and JY performed histology examination of the brain. $\mathrm{ZZ}$ and LL conducted behavioral tests. ZZ completed the data processing, normalization, and bioinformatics analyses. ZZ wrote the article with contributions from all the authors. YZ and HM supervised and fixed the writing. All authors read and approved the final manuscript.

\section{Funding}

This study was supported by the Zhongnan Hospital of Wuhan University Science, Technology and Innovation Seed Fund (WJ2019H010/znpy2018043) and by the research fund from medical Sci-Tech innovation platform of Zhongnan Hospital, Wuhan University (PTXM2020001). The funders had no role in the study design, data collection and analysis, decision to publish, or preparation of the manuscript.

\section{Availability of data and materials}

All of the datasets in this study can be obtained by reasonable request to the corresponding authors.

\section{Ethics approval and consent to participate}

All animal experimental procedures were approved by the Institutional Animal Care and Use Committee of Wuhan University.

\section{Consent for publication}

Not applicable.

\section{Competing interests}

The authors declare that they have no competing interests.

\section{Author details}

${ }^{1}$ Emergency Center, Zhongnan Hospital of Wuhan University, Wuhan 430071, China. ${ }^{2}$ Hubei Clinical Research Center for Emergency and Resuscitation, Zhongnan Hospital of Wuhan University, Wuhan 430071, China. ${ }^{3}$ Department of Biological Repositories, Zhongnan Hospital of Wuhan University, Wuhan 430071, China.

Received: 23 November 2020 Accepted: 16 January 2021 Published online: 27 January 2021

\section{References}

1. Schiller JS, Lucas JW, Ward BW, et al. Summary health statistics for U.S. adults: National Health Interview Survey, 2010. Vital Health Stat 10. 2012:1-207.

2. Cheng $P$, Yin $P$, Ning $P$, et al. Trends in traumatic brain injury mortality in China, 2006-2013: a population-based longitudinal study. PLoS Med. 2017;14:e1002332. 
3. Roozenbeek B, Maas Al, Menon DK. Changing patterns in the epidemiology of traumatic brain injury. Nat Rev Neurol. 2013;9:231-6.

4. Marin JR, Weaver MD, Mannix RC. Burden of USA hospital charges for traumatic brain injury. Brain Inj. 2017;31:24-31.

5. Leonard J, Garrett RE, Salottolo K, et al. Cerebral salt wasting after traumatic brain injury: a review of the literature. Scand J Trauma Resusc Emerg Med. 2015;23:98.

6. Cheng $\mathrm{G}$, Kong $\mathrm{RH}$, Zhang $\mathrm{LM}$, et al. Mitochondria in traumatic brain injury and mitochondrial-targeted multipotential therapeutic strategies. Br J Pharmacol. 2012;167:699-719.

7. Campos-Pires R, Hirnet T, Valeo F, et al. Xenon improves long-term cognitive function, reduces neuronal loss and chronic neuroinflammation, and improves survival after traumatic brain injury in mice. Br J Anaesth. 2019;123:60-73.

8. Tucker B, Aston J, Dines M, et al. Early brain edema is a predictor of inhospital mortality in traumatic brain injury. J Emerg Med. 2017;53:18-29.

9. Kenney K, Amyot F, Haber M, et al. Cerebral vascular injury in traumatic brain injury. Exp Neurol. 2016;275(Pt 3):353-66.

10. Carney N, Totten AM, O'Reilly C, et al. Guidelines for the management of severe traumatic brain injury, fourth edition. Neurosurgery. 2017;80:6-15.

11. Ding $H$, Wang $X$, Wang $H$, et al. Nrf2-ARE signaling provides neuroprotection in traumatic brain injury via modulation of the ubiquitin proteasome system. Neurochem Int. 2017;111:32-44.

12. Chandran R, Kim T, Mehta SL, et al. A combination antioxidant therapy to inhibit NOX2 and activate Nrf2 decreases secondary brain damage and improves functional recovery after traumatic brain injury. J Cereb Blood Flow Metab. 2018;38:1818-27.

13. Cheng T, Wang W, Li Q, et al. Cerebroprotection of flavanol (-)-epicatechin after traumatic brain injury via Nrf2-dependent and -independent pathways. Free Radic Biol Med. 2016;92:15-28.

14. Dong W, Yang B, Wang L, et al. Curcumin plays neuroprotective roles against traumatic brain injury partly via Nrf2 signaling. Toxicol Appl Pharmacol. 2018;346:28-36.

15. Chen $X$, Chen $C$, Fan $S$, et al. Omega-3 polyunsaturated fatty acid attenuates the inflammatory response by modulating microglia polarization through SIRT1-mediated deacetylation of the HMGB1/NF-KB pathway following experimental traumatic brain injury. J Neuroinflamm. 2018;15:116.

16. Mettang M, Reichel SN, Lattke M, et al. IKK2/NF-KB signaling protects neurons after traumatic brain injury. FASEB J. 2018;32:1916-32.

17. Tang $R$, Lin YM, Liu HX, et al. Neuroprotective effect of docosahexaenoic acid in rat traumatic brain injury model via regulation of TLR4/NF-Kappa B signaling pathway. Int J Biochem Cell Biol. 2018;99:64-71.

18. Yuan F, Xu ZM, Lu LY, et al. SIRT2 inhibition exacerbates neuroinflammation and blood-brain barrier disruption in experimental traumatic brain injury by enhancing NF-kB p65 acetylation and activation. J Neurochem. 2016;136:581-93.

19. $\mathrm{XuK}, \mathrm{Wu} F, \mathrm{XuK}$, et al. NaHS restores mitochondrial function and inhibits autophagy by activating the PI3K/Akt/mTOR signalling pathway to improve functional recovery after traumatic brain injury. Chem Biol Interact. 2018;286:96-105.

20. Fang J, Wang H, Zhou J, et al. Baicalin provides neuroprotection in traumatic brain injury mice model through Akt/Nrf2 pathway. Drug Des Dev Ther. 2018;12:2497-508.

21. Shen $M$, Wang $S$, Wen $X$, et al. Dexmedetomidine exerts neuroprotective effect via the activation of the PI3K/Akt/mTOR signaling pathway in rats with traumatic brain injury. Biomed Pharmacother. 2017;95:885-93.

22. Raible DJ, Frey LC, Del Angel YC, et al. JAK/STAT pathway regulation of GABAA receptor expression after differing severities of experimental TBI. Exp Neurol. 2015;271:445-56.

23. Morganti JM, Goulding DS, Van Eldik LJ. Deletion of p38a MAPK in microglia blunts trauma-induced inflammatory responses in mice. J Neuroinflamm. 2019;16:98.

24. Bhowmick S, D'Mello V, Abdul-Muneer PM. Synergistic inhibition of ERK1/2 and JNK, not p38, phosphorylation ameliorates neuronal damages after traumatic brain injury. Mol Neurobiol. 2019;56:1124-36.

25. Shim SS, Stutzmann GE. Inhibition of glycogen synthase kinase-3: an emerging target in the treatment of traumatic brain injury. J Neurotrauma. 2016;33:2065-76.

26. Lin CJ, Chen TH, Yang LY, et al. Resveratrol protects astrocytes against traumatic brain injury through inhibiting apoptotic and autophagic cell death. Cell Death Dis. 2014;5:e1147.
27. Wang $G$, Shi Y, Jiang $X$, et al. HDAC inhibition prevents white matter injury by modulating microglia/macrophage polarization through the GSK3ß/PTEN/Akt axis. Proc Natl Acad Sci USA. 2015;112:2853-8.

28. Hill JL, Kobori N, Zhao J, et al. Traumatic brain injury decreases AMPactivated protein kinase activity and pharmacological enhancement of its activity improves cognitive outcome. J Neurochem. 2016;139:106-19.

29. Cifani P, Kentsis A. Towards comprehensive and quantitative proteomics for diagnosis and therapy of human disease. Proteomics. 2017;17:1600079.

30. Smith JG, Gerszten RE. Emerging affinity-based proteomic technologies for large-scale plasma profiling in cardiovascular disease. Circulation. 2017;135:1651-64.

31. Ghazalpour A, Bennett B, Petyuk VA, et al. Comparative analysis of proteome and transcriptome variation in mouse. PLoS Genet. 2011;7:e1001393.

32. Zhang $B$, Wang J, Wang $X$, et al. Proteogenomic characterization of human colon and rectal cancer. Nature. 2014;513:382-7.

33. Liu X, Dai R, Ke M, et al. Differential proteomic analysis of dimethylnitrosamine (DMN)-induced liver fibrosis. Proteomics. 2017;17:1700267.

34. Rayner SL, Morsch M, Molloy MP, et al. Using proteomics to identify ubiquitin ligase-substrate pairs: how novel methods may unveil therapeutic targets for neurodegenerative diseases. Cell Mol Life Sci. 2019;76:2499-510

35. Drummond E, Nayak S, Faustin A, et al. Proteomic differences in amyloid plaques in rapidly progressive and sporadic Alzheimer's disease. Acta Neuropathol. 2017;133:933-54.

36. Hosp F, Gutiérrez-Ángel S, Schaefer MH, et al. Spatiotemporal proteomic profiling of Huntington's disease inclusions reveals widespread loss of protein function. Cell Rep. 2017;21:2291-303.

37. Zhang $P$, Zhang $L$, Li Y, et al. Quantitative proteomic analysis to identify differentially expressed proteins in myocardium of epilepsy using ITRAQ coupled with Nano-LC-MS/MS. J Proteome Res. 2018;17:305-14.

38. Azzam S, Schlatzer D, Nethery D, et al. Proteomic profiling of the hypothalamus in two mouse models of narcolepsy. Proteomics. 2017;17:1600478

39. Hori M, Nakamachi T, Rakwal R, et al. Transcriptomics and proteomics analyses of the PACAP38 influenced ischemic brain in permanent middle cerebral artery occlusion model mice. J Neuroinflamm. 2012;9:256.

40. Chen JH, Kuo HC, Lee KF, et al. Global proteomic analysis of brain tissues in transient ischemia brain damage in rats. Int J Mol Sci. 2015;16:11873-91.

41. Huie JR, Diaz-Arrastia R, Yue JK, et al. Testing a multivariate proteomic panel for traumatic brain injury biomarker discovery: a TRACK-TBI pilot study. J Neurotrauma. 2019;36:100-10.

42. Guingab-Cagmat JD, Newsom K, Vakulenko A, et al. In vitro MS-based proteomic analysis and absolute quantification of neuronal-glial injury biomarkers in cell culture system. Electrophoresis. 2012;33:3786-97.

43. Cheng $S X, X u Z W, Y i T L$, et al. iTRAQ-based quantitative proteomics reveals the new evidence base for traumatic brain injury treated with targeted temperature management. Neurotherapeutics. 2018;15:216-32.

44. Wu P, Zhao Y, Haidacher SJ, et al. Detection of structural and metabolic changes in traumatically injured hippocampus by quantitative differential proteomics. J Neurotrauma. 2013;30:775-88.

45. Song H, Fang S, Gao J, et al. Quantitative proteomic study reveals upregulation of CAMP signaling pathway-related proteins in mild traumatic brain injury. J Proteome Res. 2018;17:858-69.

46. Zhao L, Liu Q, Ma S, et al. TPEN attenuates neural autophagy induced by synaptically-released zinc translocation and improves histological outcomes after traumatic brain injury in rats. Ann Clin Lab Sci. 2018;48:446-52.

47. Büchele F, Morawska MM, Schreglmann SR, et al. Novel rat model of weight drop-induced closed diffuse traumatic brain injury compatible with electrophysiological recordings of vigilance states. J Neurotrauma. 2016:33:1171-80

48. Wiśniewski JR, Zougman A, Nagaraj N et al. Universal sample preparation method for proteome analysis. Nat Methods. 2009;6:359-62.

49. Li J, Donangelo I, Abe K, et al. Thyroid hormone treatment activates protective pathways in both in vivo and in vitro models of neuronal injury. Mol Cell Endocrinol. 2017:452:120-30. 
50. Sullivan PG, Sebastian AH, Hall ED. Therapeutic window analysis of the neuroprotective effects of cyclosporine A after traumatic brain injury. J Neurotrauma. 2011;28:311-8.

51. Crupi R, Paterniti I, Campolo M, et al. Exogenous T3 administration provides neuroprotection in a murine model of traumatic brain injury. Pharmacol Res. 2013;70:80-9.

52. Bareyre F, Wahl F, Mclntosh TK, et al. Time course of cerebral edema after traumatic brain injury in rats: effects of riluzole and mannitol. J Neurotrauma. 1997;14:839-49.

53. Vigil FA, Giese KP. Calcium/calmodulin-dependent kinase II and memory destabilization: a new role in memory maintenance. J Neurochem. 2018;147:12-23.

54. Zhao J, Wang T, LV Q, et al. Expression of heat shock protein 70 and Annexin A1 in serum of patients with acutely severe traumatic brain injury. Exp Ther Med. 2020;19:1896-902.

55. Wang T, Yu DR, Huang J, et al. Multimodal rehabilitation program promotes motor function recovery of rats after ischemic stroke by upregulating expressions of GAP-43, SYN, HSP70, and C-MYC. J Stroke CerebrovasC Dis. 2018;27:2829-39.

56. Gorter RP, Nutma E, Jahrei MC, et al. Heat shock proteins are differentially expressed in brain and spinal cord: implications for multiple sclerosis. Clin Exp Immunol. 2018;194:137-52.

57. Zhu Z, Reiser G. The small heat shock proteins, especially HspB4 and $\mathrm{HspB5}$ are promising protectants in neurodegenerative diseases. Neurochem Int. 2018;115:69-79.

58. Kim JY, Kim JW, Yenari MA. Heat shock protein signaling in brain ischemia and injury. Neurosci Lett. 2020;715:134642.

59. Qiu X, Ping S, Kyle M, et al. S100 calcium-binding protein A9 knockout contributes to neuroprotection and functional improvement after traumatic brain injury. J Neurotrauma. 2020;37:950-65.

60. Yang Y, Dong B, Lu J, et al. Hemopexin reduces blood-brain barrier injury and protects synaptic plasticity in cerebral ischemic rats by promoting EPCs through the HO-1 pathway. Brain Res. 2018;1699:177-85.

61. Toman E, Bishop JR, Davies DJ, et al. Vitamin D deficiency in traumatic brain injury and its relationship with severity of injury and quality of life: a prospective, observational study. J Neurotrauma. 2017;34:1448-56.

62. Gao JB, Tang WD, Wang $X$, et al. Prognostic value of neuropeptide proenkephalin $A$ in patients with severe traumatic brain injury. Peptides. 2014;58:42-6

63. Meidahl AC, Klukinov M, Tzabazis AZ, et al. Nasal application of HSV encoding human preproenkephalin blocks craniofacial pain in a rat model of traumatic brain injury. Gene Ther. 2017;24:482-6.

64. Ramesh D, Keyser-Marcus LA, Ma L, et al. Prevalence of traumatic brain injury in cocaine-dependent research volunteers. Am J Addict. 2015;24:341-7.

65. Merkel SF, Razmpour R, Lutton EM, et al. Adolescent traumatic brain injury induces chronic mesolimbic neuroinflammation with concurrent enhancement in the rewarding effects of cocaine in mice during adulthood. J Neurotrauma. 2017;34:165-81.

66. Cannella LA, McGary H, Ramirez SH. Brain interrupted: early life traumatic brain injury and addiction vulnerability. Exp Neurol. 2019;317:191-201.

67. Volkow ND, Wise RA, Baler R. The dopamine motive system: implications for drug and food addiction. Nat Rev Neurosci. 2017:18:741-52.

68. Lu K, Liang CL, Li PC, et al. Risk factors for myocardial dysfunction after traumatic brain injury: a one-year follow-up study. Injury. 2017:48:1794-800.
69. El-Menyar A, Goyal A, Latifi R, et al. Brain-heart interactions in traumatic brain injury. Cardiol Rev. 2017;25:279-88.

70. Liu YY, Brent GA. Thyroid hormone and the brain: mechisms of action in development and role in protection and promotion of recovery after brain injury. Pharmacol Ther. 2018;186:176-85.

71. Richardson SJ, Wijayagunaratne RC, D'Souza DG, et al. Transport of thyroid hormones via the choroid plexus into the brain: the roles of transthyretin and thyroid hormone transmembrane transporters. Front Neurosci. 2015;9:66.

72. Arneson D, Zhang G, Ying Z, et al. Single cell molecular alterations reveal target cells and pathways of concussive brain injury. Nat Commun. 2018;9:3894.

73. Boza-Serrano A, Ruiz R, Sanchez-Varo R, et al. Galectin-3, a novel endogenous TREM2 ligand, detrimentally regulates inflammatory response in Alzheimer's disease. Acta Neuropathol. 2019;138:251-73.

74. Siew JJ, Chen HM, Chen HY, et al. Galectin-3 is required for the microgliamediated brain inflammation in a model of Huntington's disease. Nat Commun. 2019:10:3473.

75. Nishikawa H, Nakano F, Liu L, et al. The role of galectin-3 in subarachnoid hemorrhage: a preliminary study. Acta Neurochir Suppl. 2020;127:65-8.

76. Zhai W, Chen D, Shen $\mathrm{H}$, et al. A1 adenosine receptor attenuates intracerebral hemorrhage-induced secondary brain injury in rats by activating the P38-MAPKAP2-Hsp27 pathway. Mol Brain. 2016;9:66.

77. Shi $Y$, Jiang $X$, Zhang $L$, et al. Endothelium-targeted overexpression of heat shock protein 27 ameliorates blood-brain barrier disruption after ischemic brain injury. Proc Natl Acad Sci USA. 2017;114:E1243-e1252.

78. Wilhelmsson U, Pozo-Rodrigalvarez A, Kalm M, et al. The role of GFAP and vimentin in learning and memory. Biol Chem. 2019;400:1147-56.

79. Yue JK, Yuh EL, Korley FK, et al. Association between plasma GFAP concentrations and MRI abnormalities in patients with CT-negative traumatic brain injury in the TRACK-TBI cohort: a prospective multicentre study. Lancet Neurol. 2019;18:953-61.

80. Driessen TM, Zhao C, Saenz M, et al. Down-regulation of fatty acid binding protein 7 (Fabp7) is a hallmark of the postpartum brain. J Chem Neuroanat. 2018;92:92-101.

81. Rui Q, Ni H, Lin X, et al. Astrocyte-derived fatty acid-binding protein 7 protects blood-brain barrier integrity through a caveolin-1/MMP signaling pathway following traumatic brain injury. Exp Neurol. 2019;322:113044.

82. Chirackal Manavalan AP, Kober A, Metso J, et al. Phospholipid transfer protein is expressed in cerebrovascular endothelial cells and involved in high density lipoprotein biogenesis and remodeling at the blood-brain barrier. J Biol Chem. 2014;289:4683-98.

83. Hoshi Y, Uchida Y, Kuroda T, et al. Distinct roles of ezrin, radixin and moesin in maintaining the plasma membrane localizations and functions of human blood-brain barrier transporters. J Cereb Blood Flow Metab. 2020;40:1533-45

84. Cui X, Chopp M, Zhang Z, et al. ABCA1/ApoE/HDL pathway mediates GW3965-induced neurorestoration after stroke. Stroke. 2017;48:459-67.

85. Rak M, Bénit P, Chrétien D, et al. Mitochondrial cytochrome c oxidase deficiency. Clin Sci (Lond). 2016;130:393-407.

\section{Publisher's Note}

Springer Nature remains neutral with regard to jurisdictional claims in published maps and institutional affiliations. 